\title{
Varying spread fuzzy regression for affective quality estimation
}

\author{
Kit Yan Chan, Ulrich Engelke, Senior Member IEEE
}

\begin{abstract}
Design of preferred products requires affective quality information which relates to human emotional satisfaction. However, it is expensive and time consuming to conduct a full survey to investigate affective qualities regarding all objective features of a product. Therefore, developing a prediction model is essential in order to understand affective qualities on a product. This paper proposes a novel fuzzy regression method in order to predict affective quality and estimate fuzziness in human assessment, when objective features are given. The proposed fuzzy regression also improves on traditional fuzzy regression that simulate only a single characteristic with the resulting limitation that the amount of fuzziness is linear correlated with the independent and dependent variables. The proposed method uses a varying spread to simulate nonlinear and nonsymmetrical fuzziness caused by affective quality assessment. The effectiveness of the proposed method is evaluated by two very different case studies, affective design of an electric iron and image quality assessment, which involve different amounts of data, varying fuzziness, and discrete and continuous data. The results obtained by the proposed method are compared with those obtained by the state-of-art and the recently-developed fuzzy regression methods. The results show that the proposed method can generate better prediction models in terms of three fuzzy criteria which address both predictions of magnitudes and fuzziness.
\end{abstract}

Index Terms - Fuzzy regression, varying uncertainty, affective quality, objective features, affective or perceived design, image quality assessment

\section{INTRODUCTION}

Affection quality encompasses the emotional and perceptional aesthetics of an object; it has a very significant impact on emotional satisfaction and decision-making [1]; and it is an essential process when developing pleasurable processes or products [2]. An early affective design was initialized by Kurosu and Kashimura [3] who developed two automated teller machines with identical functions. One automated teller machine had more attractive buttons and screens and the other had the unattractive ones. Survey responses elicited from users indicated that the attractive one was easier to use compared with the other. It was concluded that: products with better affective qualities work better; better affection increases purchasing chances; and it also produces a more harmonious outcome [4].

There are two different approaches to evaluate affective quality of an object: The first approach uses subjective questionnaires or surveying in order to obtain users' subjective perceptions of object aesthetics. However, it is not possible to

Kit Yan Chan is with the Department of Electrical and Computer Engineering, Curtin University, Australia; Ulrich Engelke is with Data61, Commonwealth Scientific and Industrial Research Organisation (CSIRO), Australia

(Kit Yan Chan is the corresponding author. Phone: 61-8-9266 9269; fax: 61-8-9266 7548; e-mail: kit.chan@curtin.edu.au). conduct a survey for every objective feature of a product. It is not feasible to take into account the responses from a single survey when designing and optimizing algorithms in order to enhance its affective quality. For this reason, there has been an increasing interest in automatically predicting the affective quality of an object. The second type of approaches is developed based on the assumption that users' perceptions of affective qualities are correlated with objective features such as the colour, structure, and configuration factors of the objects which can be taken into account by machine learning without involving human judgement. Objective features can be used to predict affective qualities [2]. For instance, subjective aesthetic ratings of website design are subsequently correlated with their objective features such as symmetry and balance, combination of colours and number of elements in the website [5]. Another example is image quality assessment [6]. People's opinions of an image are correlated to its objective features such as image blur and other distortions. Therefore, prediction models can be developed based on objective features in order to determine the affective quality of an object. These prediction models are generally developed by the statistical regression method as they provide explicit information such as variable significances, variable interactions and confidence intervals for dependent variables [7]. Recent research also shows that statistical regression models have been developed for the affective design of websites [5], aesthetic dental restorative materials [8], aesthetic plastic surgery [9], aesthetic evaluation of structural landscaping [10], visual aesthetics of images $[11,12]$ or video [13], and the affective design of new products [14-16] or tactile textures [17].

The statistical regression model correlates the objective features and the affective quality using a polynomial with constant coefficients. Based on a statistical regression model, the affective quality can be estimated as a crisp number, whereby the objective features of crisp numbers are given by measuring the object. However, human judgement of affective quality is subjective and therefore inherently fuzzy. Hence, fuzzy regression can be used to address the fuzziness in human judgments [18]. The fuzzy regression model is represented by a polynomial with fuzzy coefficients. The model can be used to correlate objective features in constant numbers to an affective quality in a fuzzy number. When the objective features in crisp numbers are given, the affective quality in fuzzy numbers can be estimated in order to account for the fuzziness caused by human judgement. Also, the fuzzy regression approach is more effective when only small or even incomplete data sets are available for modelling [19], since fuzzy regression does not assume that the training data is normal distributed. Research literature shows that the fuzzy regression technique has been applied to model the relationship between affective quality and objective features in order to account for the fuzziness of human 
feelings regarding car interior design [20], affective design of mobile phones [21] and image quality assessment [22].

Kao and Chyu detected a deficiency in the application of fuzzy regression models to human judgements which are subjective [23]. When the values of independent variables increase, the fuzziness of the dependent variable estimated by the models also increases. These models only simulate a single characteristic of which the amount of fuzziness is linearly correlated with the independent and dependent variables. Therefore, unnecessary fuzziness can be generated when this correlation is not linear. In fact, the fuzziness of dependent variables may decrease or remain unchanged when increasing the values of independent variables, particularly when assessing affective qualities. For example, when one evaluates the affective quality of images, one has more confidence when evaluating images of very good or very poor quality than those with medium quality [6]. Hence, more fuzziness exists when evaluating medium quality images than those with high or low qualities. Some approaches have been proposed to address this nonlinear correlation issue [23-25]. Analytical results indicate that these approaches are still unable to model the decreasing fuzziness trend of the observed dependent variables when the values of independent variables increase [26]. Although an approach [26] has been developed to address this linear correlation issue, this approach estimates the fuzziness of the dependent variables when both independent and dependent variables are fuzzy values. The approach is not developed for affective quality estimation, where the measures for independent variables, objective features, and the observations for dependent variables, affective qualities, are all crisp values. In this paper, we propose a varying spread fuzzy regression, in which the estimated fuzziness of dependent variables attempts to fit the observed variance of dependent variables. A third order polynomial is used to address the fuzziness of the dependent variables. It simulates increased, decreased or unchanged fuzziness of the affective qualities which are observed as differences between objective features. Therefore, the models are less likely to generate redundant fuzziness to describe unnecessary variances of affective quality assessment.

The effectiveness of the proposed varying spread fuzzy regression method is evaluated using two case studies, namely, affective design of an electric iron [27] and image quality assessment [28]. These two case studies involve human assessments of affective qualities, which are fuzzy. They attempt to evaluate the effectiveness of the proposed method in predicting the affective qualities of different data types including continuous data, and large and small amounts of data. The proposed method is compared with four fuzzy regression approaches: two state-of-art approaches of fuzzy regression $[18,29]$, the approach for optimizing fuzzy spreads [30, 31] and the approach developed for addressing the fuzziness increasing issue [26]. Results indicate that better prediction models can be generated by the proposed method in terms of three fuzzy criteria namely, index of confidence (IC) [32], mean fuzzy credibility (MFC) [30] and average fuzzy spread of each estimate (AFS) [18].

The rest of this paper is organized as follows: Section II presents the formulation of the affective quality model which correlates objective features and affective quality. It also discusses the limitations of the commonly-used statistical regression approaches which cannot address the fuzziness in affective quality. Section III presents the mechanisms of the existing and proposed fuzzy regression methods. It also discusses the limitations of the existing regression methods and the motivation for the proposed novel fuzzy regression method. In Section IV, two cases have been used to evaluate the effectiveness of the proposed method. Finally, a conclusion and discussion of possible future research are given in Section V.

\section{AfFective QUALITY}

Affective qualities are generally assessed based on perceptional scores from human emotional or perceptual judgement [2], where the widely used $N$-point psychometric scales usually map qualitative judgments as subjective scores [33]. People tend to judge quality around the integers with some degree of uncertainty [34]. One may refer to judgments 'about' a particular integer $X$ on psychometric scales. For instance, in image quality assessment, objective features can be directly measured as crisp values based on image distortion metrics [35] such as image blur or edge gradients. An image may be scored 'about 2' on a 10-point scale when one feels that the image quality is 'Very Poor', 'about 5' when one feels that the image quality is 'Fair' and 'about 9' when one feels that image quality is 'Very Good'. In the affective design of smartphones, objective features can be incorporated based on the design attributes of smart phones such as screen sizes, display interfaces, menu layers, shapes and colors of smartphone cases [36] all of which are catalytic data represented in crisp numbers. The affective qualities of smartphones are usually scored based on customer survey responses where high levels of affective qualities are scored when one feels the affective quality of smartphones is 'Good'. Otherwise, low levels of affective qualities are scored [37].

To predict the affective quality namely $y$, the affective quality model, $f_{A Q M}$ in (1), can be used,

$$
y=f_{\mathrm{AQM}}\left(x_{1}, x_{2}, \ldots, x_{m}\right)
$$

where $x_{j}$ with $j=1,2, \ldots, m$ is the $j$-th objective feature correlated to $y ; m$ are the number of objective features; and $f_{A Q M}$ represents the functional relationship between all $x_{j}$ to $y$. Given a set of samples of affective qualities of an object, $\left\{y(k), \bar{x}(k)^{T}\right\}$, with $k=1,2, \ldots, N_{D}, f_{A Q M}$ can be developed as a linear regression model,

$$
y=\beta_{0}+\beta_{1} x_{1}+\beta_{2} x_{2}+\ldots+\beta_{m} x_{m}+\varepsilon,
$$

where $\varepsilon$ is a random error assumed to be normally distributed with zero mean; and $\beta_{j}$ with $j=1,2, \ldots, m$ are the $m+1$ regression coefficients. $\beta_{j}$ can be estimated by the least squares method (Ch. 7 of [24]),

$$
\left[\begin{array}{llll}
\beta_{0} & \beta_{1} & \ldots & \beta_{m}
\end{array}\right]^{T}=\left(\left[\overline{1} \bar{x}^{D}\right]^{T}\left[\overline{1} \bar{x}^{D}\right]\right)^{-1}\left[\overline{1} \bar{x}^{D}\right]^{T} \bar{y}^{D}
$$

where $\overline{1}$ is the $(m+1) \times 1$ vector with all entries one; $\bar{x}^{D}=\left[\bar{x}(1) \bar{x}(2) \ldots \bar{x}\left(N_{D}\right)\right]^{T}$ is the $N_{D}$ samples of the objective features; $\bar{x}(k)=\left[x_{1}(k) x_{2}(k) \ldots x_{m}(k)\right]$ is the $m$ 
objective features of the $k$-th sample; $\bar{y}^{D}=[y(1) y(2) \ldots$ $\left.y\left(N_{D}\right)\right]^{T}$ are the affective qualities of the $N_{D}$ samples.

Given a regression model (2), one can estimate the affective quality, namely $\hat{y}_{t}$, with respect to a set of objective features on the object, namely $\bar{x}^{t}=\left(x_{1}^{t}, x_{2}^{t}, \ldots, x_{m}^{t}\right)$, where $\bar{x}^{t}$ consists of $m$ objective features $x_{i}^{t}$ with $i=1,2, \ldots, m$; and $\hat{y}_{t}$ is in a set of crisp number namely $\bar{Y}$ and $\bar{X}^{t}$ is in a $m$-dimensional vector set namely $\bar{X}$. Figure 1a illustrates that $f_{A Q M}$ performs a map between $\bar{X}$ into $\bar{Y}$ when a regression model is developed for $f_{\text {AQM }}$. For example, four vectors of objective features, $\bar{x}_{1}^{t}, \bar{x}_{2}^{t}$, $\bar{X}_{3}^{t}$ and $\bar{x}_{4}^{t}$, in $\bar{X}$ are mapped to four crisp numbers, 1.230, 3.056, 5.661 and 7.501 in $\bar{Y}$ respectively. As perception of affective quality is inherently imprecise, crisp numbers may not be the most suitable means of representing human perception of affective quality. To simulate human perception of affective qualities, $f_{A Q M}$ is essential to map $\bar{x}^{t}=\left(x_{1}^{t}, x_{2}^{t}, \ldots, x_{m}^{t}\right) \in \bar{X}$ into a linguistic term $\hat{\tilde{y}}_{t} \in \tilde{Y}$, where $\tilde{Y}$ is a set of linguistic terms with uncertainties such as the linguistic perception 'about $y^{c}$, with an integer $y^{c}$. The map, $f_{A Q M}$, can be illustrated on Figure $1 \mathrm{~b}$ that $\bar{X}$ is mapped into $\tilde{Y}$. Through $f_{A Q M}, \bar{x}_{1}^{t}, \bar{X}_{2}^{t}$, $\bar{X}_{3}^{t}$ and $\bar{x}_{4}^{t}$, in $\bar{X}$ can be mapped to the four linguistic terms, 'about 1', 'about 3', 'about 5', 'about 7' in $\tilde{Y}$ respectively.

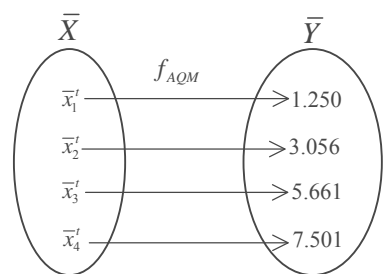

$f_{A Q M}$ maps between crisp real values in $\bar{X}$ to crisp real values in $\bar{Y}$

Fig. 1a Mapping to crisp values Fig. 1b Mapping to linguistic terms

In fuzzy theory, the linguistic term 'about $y^{c}$ ' can be considered as an ill-known truth-value with uncertainty of gradual truth at the crisp value $y^{c}$ [38]. 'about $y^{c}$ ' can be explained by a fuzzy number, $\tilde{y}=\left(y^{c}, y^{s}\right)$, which is engaged with a fuzzy membership function, $\mu_{\tilde{y}}(y)$ [34] in (4). A triangle function is used in (4), as it requires less complicated fuzzy arithmetical operations compared with Gaussian or trapezoidal functions [39]. Given a quantity value $y$, the membership grade of 'about $y^{c}$ can be determined by $\mu_{\tilde{y}}(y)$.

$$
\mu_{\tilde{y}}(y)= \begin{cases}1 & y=y^{c} \\ 1-\frac{\left|y-y^{c}\right|}{y^{s}} & y^{c}-y^{s}<y<y^{c}+y^{s} \\ 0 & \text { otherwise }\end{cases}
$$

In $\mu_{\tilde{y}}(y)$, a full membership grade of the linguistic term 'about $y^{c}$ ' can be granted when $y=y^{c}$ is given. Given that $y^{c}-y^{s}<y<y^{c}+y^{s}, y$ has a membership grade with 'about $y^{c}$ '. When $y$ is not within the range between $\left(y^{c}-y^{s}\right)$ and $\left(y^{c}+y^{s}\right), y$ has no membership with 'about $y^{c} \cdot y^{c}$ is the center of $\tilde{y} . y^{s}$ is the spread of $\tilde{y}$, which indicates the fuzziness of human evaluation of affective qualities. As an illustration, Figure 2 shows three fuzzy numbers, 'about 2' $\tilde{y}_{1}=(2,1)$, 'about 8 ' $\tilde{y}_{2}=(8.3)$, and 'about 5 ' $\tilde{y}_{3}=(5,2)$, on a 10 -point scale for affective quality evaluation. The fuzziness of $\tilde{y}_{3}$ is less than $\tilde{y}_{2}$ but is more than $\tilde{y}_{1}$.

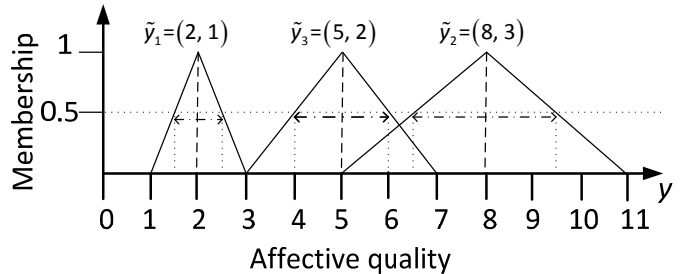

Fig. 2 The affective qualities in fuzzy numbers

\section{FUZZY REGRESSION FOR AFFECTIVE QUALITY} A. Fuzzy regression with linear spreads

Given a set of objective features, $\bar{x}=\left(x_{1}, x_{2}, \ldots, x_{m}\right)$, the fuzzy regression model with symmetrical coefficient spreads [40], $\tilde{F}_{\text {Lin }}(\bar{x})=\left(f_{\text {Lin }}^{C}(\bar{x}), f_{\text {Lin }}^{S}(\bar{x})\right)$ in $(5 \mathrm{a})$, can be used to determine the corresponding affective quality in a fuzzy number $\hat{\tilde{y}}=\left(\hat{y}^{C}, \hat{y}^{S}\right)$ with symmetrical spreads.

$$
\hat{\tilde{y}}=\tilde{F}_{\text {Lin }}(\bar{x})=\left(f_{\text {Lin }}^{C}(\bar{x}), f_{\text {Lin }}^{S}(\bar{x})\right)=\tilde{A}_{0}+\tilde{A}_{1} x_{1}+\cdots \tilde{A}_{j} x_{j} \cdots+\tilde{A}_{m} x_{m}
$$

where $\hat{y}^{\gamma}=f_{\text {Lin }}^{\gamma}(\bar{x})=a_{0}^{\gamma}+a_{1}^{\gamma} X_{1}+\cdots a_{j}^{\gamma} x_{j} \cdots+a_{m}^{\gamma} x_{m}$ with $\gamma=C$ or $\gamma=S$.

$\hat{y}^{C}\left(\right.$ or $\left.f_{\text {Lin }}^{C}(\bar{x})\right)$ and $\hat{y}^{S}\left(\right.$ or $\left.f_{\text {Lin }}^{S}(\bar{x})\right)$ address the quantity and uncertainty of evaluating affective quality with respect to $\bar{x}$ respectively; and $\tilde{A}_{j}$ with $j=1,2, \cdots, m$ are the fuzzy coefficients which are given by $\tilde{A}_{j}=\left(a_{j}^{C}, a_{j}^{S}\right)$, with the central $a_{j}^{C}$ and the spread $a_{j}^{s}$. All $\tilde{A}_{j}$ in (5a) correlate $\bar{x}$ to $\hat{\tilde{y}}$. $\tilde{A}_{j}$ in the fuzzy regression model (5a) are not as sharply defined as the regression coefficients $\beta_{j}$ in the non-fuzzy regression model (2) of which all $\beta_{j}$ are crisp numbers [18]. All $a_{j}^{s}$ in $\tilde{A}_{j}$ address the deviations between the estimated to the observed affective qualities. Hence, $a_{j}^{s}$ indicates the amount of fuzziness caused by the human judgement [18]. The non-fuzzy regression (2) addresses the deviation $\varepsilon$ which is assumed to be normally distributed with zero mean [41]. To satisfy this normal distribution assumption, a large set of samples is required to develop a non-fuzzy regression model. The processing of large data sets is computationally expensive. Hence, there is a trade-off between the accuracy of the non-fuzzy regression estimate and the cost of data collection [40].

$\tilde{F}_{L i n}(\bar{x})$ in $(5 \mathrm{a})$ can be used to estimate, $\hat{\tilde{y}}=\left(\hat{y}^{C}, \hat{y}^{S}\right)$, engaging with $\mu_{\tilde{y}}(y)$ in (4), where $\hat{y}^{C}$ and $\hat{y}^{S}$ can be determined based on (5b). The fuzzy regression model achieves 
two goals [51]: First, the fuzzy regression model attempts to satisfy the constraint (6) for each sample $\left\{y(k), \bar{x}(k)^{T}\right\}$,

$$
\mu_{\tilde{y}}(y(k)) \geq 1-h \text { for all } k=1,2, \ldots, N_{D}
$$

(6) ensures that the model estimates can grant a membership grade with more than $h$, to all $y(k)$, where $h$ is specified by the decision maker. Second, the fuzzy regression model attempts to minimize the overall estimate of fuzziness $Z=\sum_{i=1}^{N_{D}} f_{\text {Lin }}^{s}(\bar{x}(i))$, which is the total fuzziness with respect to all $\bar{x}(i)$. Hence, the constrained optimization problem (7a) to (7d) [18] is formulated in order to determine $\tilde{A}_{j}=\left(a_{j}^{C}, a_{j}^{S}\right) \quad$ with $j=1,2, \cdots, m$, in (5a) and (5b):

$$
\begin{aligned}
& \min Z=\sum_{i=1}^{N_{D}} f_{\text {Lin }}^{S}(\bar{x}(i)), \text { subject to } \\
& h f_{\text {Lin }}^{S}(\bar{x}(i))-f_{\text {Lin }}^{C}(\bar{x}(i)) \geq-y(i), i=1,2, \ldots, N_{D}, \\
& h f_{\text {Lin }}^{S}(\bar{x}(i))+f_{\text {Lin }}^{C}(\bar{x}(i)) \geq y(i), i=1,2, \ldots, N_{D} ; \\
& a_{j}^{\mathrm{S}} \geq 0, j=0,1, \cdots, m
\end{aligned}
$$

where $f_{\text {Lin }}^{C}(\bar{x}(i))$ and $f_{\text {Lin }}^{S}(\bar{x}(i))$ represent the central and spread of estimated affective quality $\tilde{F}_{\text {Lin }}(\bar{x}(i))=$ $\left(f_{\text {Lin }}^{C}(\bar{x}(i)), f_{\text {Lin }}^{S}(\bar{x}(i))\right)$ with respect to $\bar{x}(i)$ respectively; $f_{\text {Lin }}^{C}(\bar{x}(i))$ and $f_{\text {Lin }}^{S}(\bar{x}(i))$ are given by $(8)$ :

$$
f_{\text {Lin }}^{\gamma}(\bar{x}(i))=a_{0}^{\gamma}+\sum_{j=1}^{m} a_{j}^{\gamma} x_{j}(i), \text { with } \gamma=C \text { or } \gamma=S .
$$

The last constraint (7d) ensures that all $a_{j}^{\mathrm{s}}$ are non-negative. When $a_{j}^{\mathrm{s}}$ and all elements in $\bar{x}(i)$ are positive, $f_{\text {Lin }}^{\mathrm{s}}(\bar{x}(i))$ can be ensured to be positive. Figure 3 a illustrates how the fuzzy regression model satisfies the constraints (7b) and (7c), and the amount of overall fuzziness $Z$ exists in the model. The constraints are illustrated by the $h$-interval between $\left(f_{\text {Lin }}^{C}(\bar{x}(i))+h \cdot f_{\text {Lin }}^{S}(\bar{x}(i))\right)$ and $\left(f_{\text {Lin }}^{C}(\bar{x}(i))-h \cdot f_{\text {Lin }}^{S}(\bar{x}(i))\right)$ which bounds all samples $y(i)$. Hence, the membership grade of each $y(i)$ is larger than $h$ under the fuzzy estimates $\tilde{F}_{\text {Lin }}(\bar{x}(i))=\left(f_{\text {Lin }}^{C}(\bar{x}(i)), f_{\text {Lin }}^{S}(\bar{x}(i))\right)$ which is generated by the fuzzy regression model (5a) with respect to $\bar{x}(i) . Z$ is illustrated by the fuzzy interval between $\left(f_{\text {Lin }}^{C}(\bar{x}(i))+f_{\text {Lin }}^{S}(\bar{x}(i))\right)$ and $\left(f_{\text {Lin }}^{C}(\bar{x}(i))-f_{\text {Lin }}^{S}(\bar{x}(i))\right)$. Given a specified set of objective features, $\bar{x}^{t}=\left(x_{1}^{t}, x_{2}^{t}, \ldots, x_{m}^{t}\right)$, Figure 3a shows how the affective quality in fuzzy number $\hat{\tilde{y}}^{t}=\left(y_{t}^{C}, y_{t}^{S}\right)$, is estimated by the fuzzy regression model, where $y_{t}^{C}$ is the center and $y_{t}^{S}$ is the fuzzy spread. The view vision of $\hat{\tilde{y}}^{t}$ is illustrated by Figure $3 \mathrm{~b}$. All $y(i)$ are covered by the $h$-interval between $\left(y_{t}^{C}+h \cdot y_{t}^{S}\right)$ and $\left(y_{t}^{C}-h \cdot y_{t}^{S}\right)$.
The overall fuzziness of the model is indicated by the width of the fuzzy interval, $2 \cdot\left|f_{\text {Lin }}^{S}(\bar{x})\right|$, given in $(5 b)$ which correlates linearly with the objective features $\bar{x}=\left[x_{1}, x_{2}, \ldots, x_{m}\right]$. $2 \cdot\left|f_{\text {Lin }}^{S}(\bar{x})\right|$, increases when the magnitudes of $\bar{x}$ increase, and thus the estimated overall fuzziness increases when increasing the objective features. We consider a simple model with one objective feature (i.e. $\bar{x}=\left[x_{1}\right]$ ) correlated to the affective quality $\hat{\tilde{y}}$. The spread of the simple model, $f_{\text {Lin }}^{s}(\bar{x})$, is given in (9), where $a_{0}^{s}$ and $a_{1}^{s}$ represent the coefficient spreads. It shows clearly that $2 \cdot\left|f_{\text {Lin }}^{S}(\bar{x})\right|$, increases when $x_{1}$ increases.

$$
f_{\text {Lin }}^{S}(\bar{x})=a_{0}^{S}+a_{1}^{S} x_{1}
$$

Figure 3 a shows that the variances of affective qualities are smaller when $\bar{x}$ is either small or large. It shows that variances of the affective qualities are greater when $\bar{x}$ is in the median. Hence, little fuzziness exists when the objective features are at the two extremes. In real-life situations, the fuzziness of observed dependent variables may decrease or remain unchanged when the magnitudes of objective features increase [26]. More fuzziness exists in the medium objective feature as people are more uncertain when evaluating medium affective quality. Also, the fuzziness may not be symmetrical as generated by the fuzzy regression [42]. Therefore, unnecessary fuzziness can be estimated in fuzzy regressions with linear spreads (8) when $x_{1}$ increases. This is the deficiency of fuzzy regressions with linear spreads which may inaccurately estimate the fuzziness of evaluating affective quality [26].

\section{B. Proposed fuzzy regression with varying spread}

In this paper, a novel fuzzy regression approach involving varying spreads is proposed in order to address the nonlinear and nonsymmetrical fuzziness of affective qualities, where the spreads are developed in cubic polynomial form as illustrated in Figure 4 . The varying spreads of the proposed fuzzy regression model are illustrated in Figure 4. The proposed model in a triplet is given as follows:

$$
\hat{\tilde{y}}=\tilde{F}_{V S}(\bar{x})=\left(f_{V S}^{C}(\bar{x}), f_{V S}^{L}(\bar{x}), f_{V S}^{R}(\bar{x})\right)
$$

where $f_{V S}^{C}(\bar{x})$ is the central; $f_{V S}^{L}(\bar{x})$ is the left spread; and $f_{V S}^{R}(\bar{x})$ is the right spread. The proposed model in (10) is developed based on a two-phase methodology whereby the central $f_{V S}^{C}(\bar{x})$ is determined in the first phase and the two spreads, $f_{V S}^{L}(\bar{x})$ and $f_{V S}^{R}(\bar{x})$, are determined in the second phase. In the first phase, $f_{V S}^{C}(\bar{x})$ is developed in the form as (5b) with $\gamma=C$, where the centrals of the fuzzy coefficients, $a_{i}^{C}$ with $i=1,2, \ldots, m$, are computed by the ordinary least square regression [29]. All $a_{i}^{C}$ with $i=1,2, \ldots, m$ are determined by:

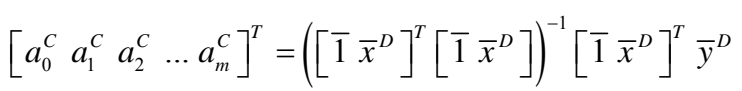

where $\overline{1}$ is the $(m+1) \times 1$ vector with all one elements. $f_{V S}^{L}(\bar{x})$ and $f_{V S}^{R}(\bar{x})$ are formulated by the polynomials with three orders [42] respectively as: 


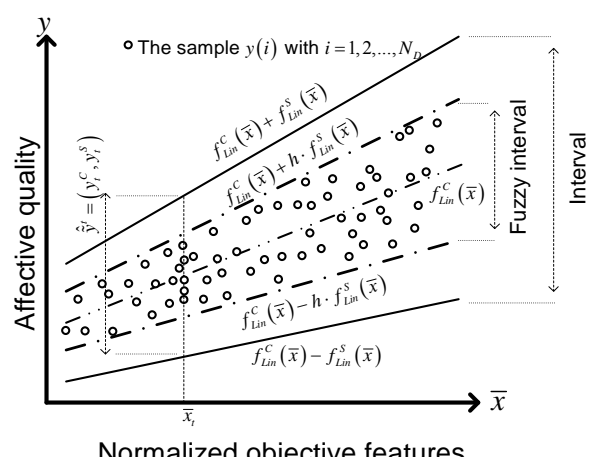

Normalized objective features

Fig. 3a Fuzzy regression model for estimating affective quality, $\tilde{F}_{\text {Lin }}(\bar{x})=\left(f_{\text {Lin }}^{C}(\bar{x}), f_{\text {Lin }}^{S}(\bar{x})\right)$

$$
f_{V S}^{L}(\bar{x})=\sum_{j=1}^{m}\left(a_{j, 0}^{L}+a_{j, 1}^{L} \cdot x_{j}+a_{j, 2}^{L} \cdot\left(x_{j}\right)^{2}+a_{j, 3}^{L} \cdot\left(x_{j}\right)^{3}\right),
$$

and $f_{V S}^{R}(\bar{x})=\sum_{j=1}^{m}\left(a_{j, 0}^{R}+a_{j, 1}^{R} \cdot x_{j}+a_{j, 2}^{R} \cdot\left(x_{j}\right)^{2}+a_{j, 3}^{R} \cdot\left(x_{j}\right)^{3}\right)$

Unlike the spreads formulated in (5b) with $\gamma=S$ which consists only the linear polynomials, the proposed spreads, $f_{V S}^{L}(\bar{x})$ in (12a) and $f_{v S}^{R}(\bar{x})$ in (12b) are involved with three order terms for the objective features. $f_{V S}^{L}(\bar{x})$ and $f_{V S}^{R}(\bar{x})$ attempt to model the fuzziness which is not necessary to be linear to $\bar{x} . f_{V S}^{L}(\bar{x})$ and $f_{V S}^{R}(\bar{x})$ take into account possible nonlinearity between $\bar{x}$ to fuzziness [42]. It attempts to overcome the limitation of the existing fuzzy regression that only models one characteristic that the fuzziness is linearly related to the objective features.

The second phase minimizes the overall fuzziness $\left|f_{V S}^{R}(\bar{x})-f_{V S}^{L}(\bar{x})\right|$ and ensures that the estimates can grant more than $h$ membership to all affective quality samples, $y(k)$. We determine all $a_{0}^{L}, a_{0}^{R}, a_{j, i}^{L}$ and $a_{j, i}^{R}$ with $j=1,2 .,,, m$ and $i=$ $1,2,3$ in $f_{V S}^{L}(\bar{x})$ and $f_{V S}^{R}(\bar{x})$ by solving the optimization problem (13a-e) with respect to the sample set $\left\{y(k), \bar{x}(k)^{T}\right\}$ with $k=1,2, \ldots, N_{D}$ :

$$
Z=\min \sum_{k=1}^{N_{D}}\left(f_{V S}^{R}(\bar{x}(k))+f_{V S}^{L}(\bar{x}(k))\right)
$$

subject to $h f_{V S}^{L}(\bar{x}(k))-f_{V S}^{C}(\bar{x}(k)) \geq-y(k)$;

$$
\begin{aligned}
& h f_{V S}^{R}(\bar{x}(k))+f_{V S}^{C}(\bar{x}(k)) \geq y(k) ; k=1,2, \ldots, N_{D} \\
& f_{V S}^{L}(\bar{x}(k)) \geq 0, f_{V S}^{R}(\bar{x}(k)) \geq 0 \quad k=1,2, \cdots, N_{D}
\end{aligned}
$$

where $f_{V S}^{C}(\bar{x}(k)), f_{V S}^{L}(\bar{x}(k))$ and $f_{V S}^{R}(\bar{x}(k))$ are the central, left spread and right spread with respect to the $k$-th object feature sample, $\bar{x}(k)$. They are given respectively as:

$$
\begin{aligned}
f_{V S}^{\gamma}(\bar{x}(k))= & \sum_{j=1}^{m}\left(a_{j, 1}^{\gamma} \cdot x_{j}(k)+a_{j, 2}^{\gamma} \cdot\left(x_{j}(k)\right)^{2}+a_{j, 3}^{\gamma} \cdot\left(x_{j}(k)\right)^{3}\right)+a_{0}^{\gamma} \\
& +a_{0}^{\gamma} \text { with } \gamma=R \text { or } \gamma=L
\end{aligned}
$$

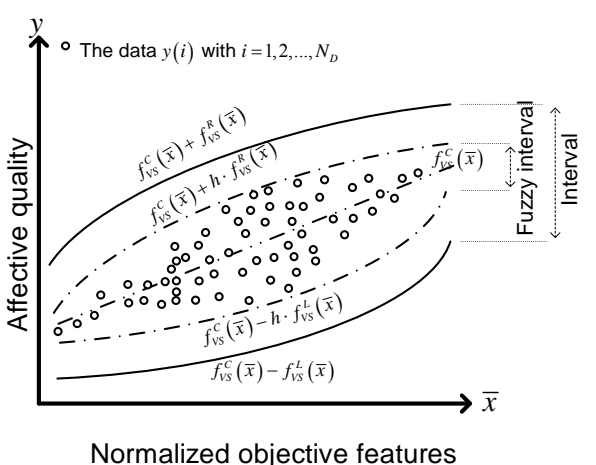

Fig. 4 Fuzzy regression model with polynomial spreads $\tilde{F}_{V S}(\bar{x})=\left(f_{v S}^{C}(\bar{x}), f_{v S}^{L}(\bar{x}), f_{V S}^{R}(\bar{x})\right)$

$$
f_{v S}^{C}(\bar{x}(k))=a_{0}^{C}+\sum_{j=1}^{m} a_{j}^{C} \cdot x_{j}(k)
$$

Given a specified membership grade $h$, the fuzzy interval of the model is denoted as $\left(f_{V S}^{C}(\bar{x}(k))-h \cdot f_{V S}^{L}(\bar{x}(k)), f_{V S}^{C}(\bar{x}(k))\right.$ $\left.+h \cdot f_{V S}^{R}(\bar{x}(k))\right)$, where all samples received a higher than $h$ membership grade from the model. The fuzzy interval covers all $y(k)$ using the polynomial spreads (13d). Based on the polynomial spreads, unnecessary fuzziness is less likely to be generated than those generated by the fuzzy regression with linear spreads, formulated in (5a) and (5b). Both classical and heuristic optimization methods namely VS-FR-SM and VS-FR-GA are proposed to generate the fuzzy regression (10) by solving the optimization problem (13a-e). Both VS-FR-SM and VS-FR-GA attempt to determine the optimal fuzzy spread coefficients $a_{0}^{L}, a_{0}^{R}, a_{j, i}^{L}$ and $a_{j, i}^{R}$ with $j=1,2 .,,, m$ and $i=$ $1,2,3$, after the center coefficients $a_{j}^{C}$ with $j=0,1,2 .,,, m$ in (11) are predetermined using the ordinary least square regression. In VS-FR-SM, the simplex method is used to determine the fuzzy spread parameters by solving the optimization problem consists of the cost function formulated in (13a) and the constraints formulated in (13b) to (13e). The simplex method is proposed as it has commonly been used on developing fuzzy regressions [43]. In VS-FR-GA, the genetic algorithm is used to determine the fuzzy spread parameters, as it is not susceptible to a lack of convexity of the solution landscapes [44] and also it can determine better fuzzy coefficients for generating more accurate models, compared with the simplex method [45]. The framework of the VS-FR-SM and VS-FR-GA are summarized in Figure 5.

Both VS-FR-SM and VS-FR-GA are implemented using Matlab 7.7 in a PC which has a CPU of Intel(R) Core(TM)2 Duo $2.66 \mathrm{GHz}$ and a memory of $8 \mathrm{~GB}$. The development of novel optimization method is not the main contribution of this research. Hence, the default parameters for the simpler method are used in the VS-FR-SM. The VS-FR-GA is implemented by the ga solver in the global optimization toolbox in Matlab, which is effective to handle linear and bound constraints. 


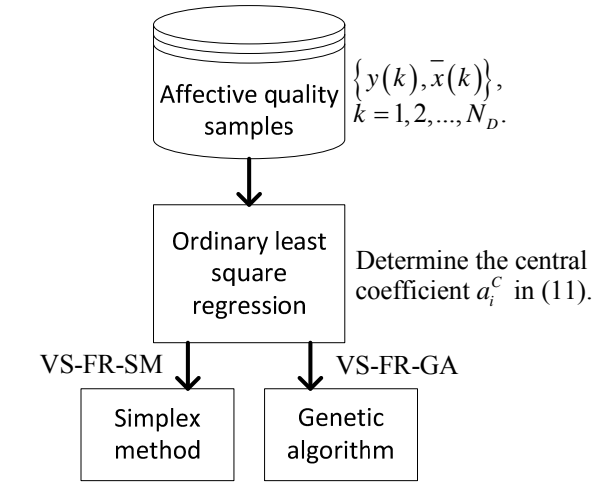

Determine the spread coefficients, $a_{j, 1}^{L}, a_{j, 2}^{L}, a_{j, 3}^{L}$, $a_{j, 1}^{R}, a_{j, 2}^{R}, a_{j, 3}^{R}$ in (12a) and (12b).

Fig. 5 Framework of VS-FR-SM and VS-FR-GA

In the ga solver, the chromosomes are represented as the fuzzy spread coefficients $a_{0}^{L}, a_{0}^{R}, a_{j, i}^{L}$ and $a_{j, i}^{R}$ with $j=1,2$, $\ldots, m$ and $i=1,2,3$. The ga solver first randomly generates a population of chromosomes which are in real numbers. Each gene in the chromosome represents a fuzzy spread coefficient. Hence, the number of genes in a chromosome is given as $N_{v}=4 \times 3 \times m=12 m$. The population is evolved iteratively based on the two operations, mutation and crossover. For the mutation, a random number is added to a selected gene, where the random number is generated with Gaussian distribution with mean zero and it needs to satisfy the constraints (13b) to (13e), as the ga solver attempts to solve the constrained optimization problem. For the crossover, the intermediate operation is used. The crossover generates the children by averaging the parents with a random weight. For example, two parents, $\bar{p}_{1}$ and $\bar{p}_{2}$ are selected to perform the crossover. A child is generated as, $\bar{c}=\bar{p}_{1}+r \times\left(\bar{p}_{1}-\bar{p}_{2}\right)$, where $r$ is a random number between 0 to 1 . The ga solver uses the mutation and crossover operations to generate feasible solutions which satisfy the constraints in (13b) to (13e). After the feasible solution is found, the ga solver uses the approach of roulette-wheel to select the good chromosomes with respect to the cost function formulated by (13a). The ga solver attempts to find a better feasible solution with a smaller spread. The ga solver goes to the next evolutionary generation until the termination condition is met. In the ga solver, the following parameters [46] are used: crossover rate $=0.8$; mutation rate $=1 / N_{v}$; total generations $=1000$; population size $=200$. The ga solver keeps evolving the chromosomes until the fitness of the chromosomes cannot be improved within 10 generations.

\section{CASE STUDIES FOR AFFECTIVE QUALITY MODELLING}

Two case studies, the affective design of an electric iron [27] and image quality assessment [28], have been conducted in order to evaluate the effectiveness of the proposed fuzzy regression methods: one using the simplex method Table 1 Data characteristics of the two case studies.
(VS-FR-SM) and the other using the genetic algorithm (VS-FR-GA). These two case studies involve subjective human evaluations of affective qualities. They also simulate the different characteristics of affective designs. The affective design of an electric iron involves categorical data for objective features and the image quality assessment involves continuous ones. Also, the amount of data involved in the two case studies is very different. Only a small data set is used for the affective design of the electric iron as only 8 samples are involved. It attempts to simulate the practical situation where only small samples are available for affective design. A much larger amount of data (1725 samples) is involved in the image quality assessment case study. Hence, the effectiveness of the proposed methods can be evaluated for different data sizes. Table 1 summarizes the data characteristics of the two case studies.

The proposed methods are compared with the state-of-art and recently-developed fuzzy regression methods. The Tanaka fuzzy regression approach, namely TS-FR [18] is considered, where TS-FR is the first fuzzy regression approach which is formulated by minimizing the fuzzy spreads in linear polynomials. Also, the fuzzy regression integrated with statistical regression, namely TS-SR-FR, is considered [29], that uses the statistical regression to determine the center of the model. TS-SR-FR is similar to the proposed VS-FR-SM and VS-FR-GA which all use statistical regression to determine the center; however, linear spreads are used in TS-SR-FR while varying spreads are used in the proposed approaches. This comparison demonstrates the effect of using linear spreads and varying spreads on the fuzzy regression. To further validate the effectiveness, the proposed approaches are compared with a recent fuzzy regression method, namely FC-FR [30, 31], which determines the fuzzy spreads by maximizing the fuzzy credibility of the model.

In the statistical regression, goodness-of-fitness is usually used in order to evaluate how effective the model can fit the samples. Goodness-of-fitness indicates the similarity between the samples and the estimates generated by the model. Based on the goodness-of-fitness, performance of models can be indicated. Unlike statistical regression models which only consists of a single central, fuzzy regression models consists of the central and the interval which consists of the right and left spreads. Statistical regression models only attempt to predict crisp values for dependent variables. Goodness-of-fitness used for evaluating statistical regression model is not the most appropriate to evaluate fuzzy regression, as the goodness-of-fitness only addresses the central of model and it cannot interpret the interval of the fuzzy regression model. Difference performance measures are used to evaluate the intervals of fuzzy regression models. The following three fuzzy criteria are used to evaluate the performance of these fuzzy regression approaches:

\begin{tabular}{|l|l|l|}
\hline & electric iron design & Image quality assessment \\
\hline Number of samples & 8 & 1725 \\
\hline Data type of objective features & Categorical & Continuous \\
\hline Number of objective features & 5 & 17 \\
\hline Number of affective quality assessments & 40 & 256428 \\
\hline
\end{tabular}


1. The proposed and the existing fuzzy regression approaches attempt to use an interval to cover all the samples where the interval is constrained by bounding all samples within the interval. All fuzzy regression approaches attempt to minimize the interval width in order to cover all the samples. As all fuzzy regression approaches attempt to generate a small interval, they attempt to generate a model with a small average fuzzy spread of each estimate (AFS) [18] which is given in (14). The AFS can be used to evaluate the interval size. When the interval is small, the model is better.

$$
\mathrm{AFS}=\sum_{k=1}^{N_{D}} h \cdot \Delta \hat{\tilde{y}}(k)=\sum_{k=1}^{N_{D}} h \cdot\left|y^{R}(k)-y^{L}(k)\right|
$$

AFS also evaluates the overall fuzziness generated by the model. When AFS is smaller, unnecessary fuzziness is less likely to be generated by the fuzzy regression model. However, AFS only takes into account the interval of the model but it cannot address the central. Despite AFS, the following two fuzzy criteria are used to address both the interval and the central of the model.

2. Index of confidence (IC) [32] is similar to the goodness-of-fitness $R^{2}$ in statistical regression which measures how close the samples are to the fitted regression line. The IC measures how close the samples are to the central and the interval of the fuzzy regression. The IC is given by the degree of variation of the samples $y(k)$ to the intervals of the fuzzy estimates $\hat{\tilde{y}}(k)=\left(\hat{y}^{C}(k), \hat{y}^{L}(k)\right.$ $\left.\hat{y}^{R}(k)\right)$ with $k=1,2, \ldots, N_{D}$, where $\hat{\tilde{y}}(k)$ is generated by the fuzzy regression model. The IC is given by:

$$
\mathrm{IC}=\frac{\mathrm{SSR}}{\mathrm{SST}} \text {, }
$$

where SST represents the total variation of $y(k)$ to the left spread, $\hat{y}^{L}(k)$, and right spread, $\hat{y}^{R}(k)$, of the fuzzy estimate and SSR represents the variation of center $y^{C}(k)$ of the fuzzy estimates to both $\hat{y}^{L}(k)$ and $\hat{y}^{R}(k)$. SST and SSR are given by,

$$
\begin{aligned}
& \mathrm{SST}=\sum_{k=1}^{N_{D}}\left(y(k)-\hat{y}^{L}(k)\right)^{2}+\sum_{k=1}^{N_{D}}\left(y(k)-\hat{y}^{R}(k)\right)^{2} ; \\
& \mathrm{SSR}=\sum_{k=1}^{N_{D}}\left(\hat{y}^{C}(k)-\hat{y}^{L}(k)\right)^{2}+\sum_{k=1}^{N_{D}}\left(\hat{y}^{C}(k)-\hat{y}^{R}(k)\right)^{2}
\end{aligned}
$$

When IC is larger, the value of SSR is closer to SST, and the estimates generated by the fuzzy regression model are a better fit to the samples. Hence, the fuzzy regression model is better when its IC is larger. Based on the IC, the central and the interval of the model can be taken into account. IC has been used for evaluating fuzzy regression models for new product development [27, 47] and management satisfaction [32].

3. Mean fuzzy credibility (MFC) [30] has recently been developed to evaluate fuzzy regression performance. The MFC evaluates the memberships of the collected data with respect to the fuzzy regression model and the overall fuzziness of the model. The MFC is given as:

$$
\mathrm{MFC}=\sum_{k=1}^{N_{D}} \frac{\mu_{\hat{y}}(y(k))}{\Delta(\hat{\tilde{y}}(k))}=\sum_{k=1}^{N_{D}} \frac{\mu_{\hat{y}}(y(k))}{\left|y^{R}(k)-y^{L}(k)\right|}
$$

The denominator indicates the amount of fuzzy spreads which represent the fuzziness of the estimates; the numerator indicates the fuzzy membership of the samples with respect to the model. When the MFC is higher, the denominator is high and the numerator is low. The fuzzy regression model can generate higher memberships to sample and generate estimates with less fuzziness. Unnecessary fuzziness is less likely to be generated and better membership can be given by the model when a higher MFC is obtained. MFC has been used to evaluate fuzzy regression models for new product development [31] and manufacturing processes [30].

\section{A. Affective design of electric iron}

In the affective design of an electric iron, its attractiveness is considered as the affective quality. We develop the models which correlate the attractiveness to the objective features of electric irons, where the iron shape, colour and components are considered as the objective features which evoke a response to the attractiveness of electric irons. Based on the models, the attractiveness of the electric iron can be estimated and optimized with respect to the objective features [14]. Electric irons with good affective quality can attract customers and influence their choice of product.

1) Data description

Prior to develop models, a survey was conducted based on a competitive benchmark for eight electric irons [27]. Figure 6 in the appendix shows the objective features of electric irons including body color tone $x_{1}$, soleplate $x_{2}$, handle design $x_{3}$, spray button $x_{4}$, and water level indicator $x_{5}$. The morphological description of the electric irons shown in Table 2 shows the five features: $x_{1}$ is either warm or cold tone; $x_{2}$ is either sharp or round tip; $x_{3}$ is either embedded, ' 7 ', ' $\Gamma$ ', or ' $\mathrm{T}$ ' shape; $x_{4}$ is either flat, curvy or handle shape; and $x_{5}$ is either transparent or sandblasted.

These objective features are correlated to the affective quality of an electric iron. Table 2 in the appendix shows the quantitative values of the affective features which are transformed to categorical data. As an example, Electric Iron A consists of $x_{1}$ with warm tone color, $x_{2}$ with sharp tip soleplate, $x_{3}$ with embedded type handle, $x_{4}$ with flat button, and $x_{5}$ with transparent indicator. $x_{1}$ consists of two categories and thus it can be transformed as 0.50 and 1.00. $x_{3}$ consists of four categories and thus it can be transformed as $0.25,0.50$, 0.75 and 1.00. Similarly, the rest of the objective features can be transformed as qualitative values based on the number of categories. As eight electric irons are benchmarked, this case study is involved with eight samples which are illustrated in Table 3 in the appendix. To assess the affective qualities of the electric irons, a survey was conducted by five interviewees, who have more than 15 years of experience in using electric irons and also have experience in purchasing more than 3 electric irons. These interviewees assessed the attractiveness of each electric iron, using scores from 1 to 4 . The last row of Table 3 shows the average attractiveness of each electric iron, $y$, which is the mean of the five interviewees' scores. As there are eight electric irons and five interviewees, forty affective quality assessments have been conducted. 
In new product development, this is time consuming to perform customer surveys. Also the number of products in the market is limited. Hence, small number of samples is generally available to develop prediction models for product analysis. Fuzzy regression is particularly effective to develop models when a small number of samples is only available [40]. For example, 10 samples were used for developing fuzzy regression models for mobile phones design [36]; 5 samples were used for developing models for car products [48]; 5 samples were used for developing models for dynamite packing machine [49]. Although this case study of electric iron design is involved only with 8 samples, existing research [27] has been conducted on developing fuzzy regression models using the classical Tanaka approach (TS-FR) [18]. Therefore, the proposed approach can be used to further improve the modelling performance. Through this case study, the effectiveness of the proposed approach can be evaluated when a small set of samples is given.

2) Numerical results

Table 4 shows the fuzzy regression models developed by the four existing methods, TS-FR, TS-SR-FR, FC-FR and LW-FR, and the proposed methods, VS-FR-SM and VS-FR-SM. These fuzzy regression models can be used to estimate the crisp values and fuzziness of the attractiveness of the electric iron, when the objective features are given. Due to space limitations, we illustrate only those models represented by the body color tone $x_{1}$ and the spray button design $x_{4}$ which have different number of categorical levels.

Figures 7 illustrates the fuzzy regression models which correlate $x_{1}$ to the attractiveness $y$, where Figures $7 \mathrm{a}, 7 \mathrm{~b}, 7 \mathrm{c}$, $7 \mathrm{~d}, 7 \mathrm{e}$ and $7 \mathrm{f}$ show the models generated by TS-FR, TS-SR-FR, FC-FR, LW-FR, VS-FR-SM and VS-FR-SM respectively. In these figures, the solid lines show the centrals and the fuzzy intervals of the models and the dotted lines show the $h$-intervals of the models, where all the collected data is constrained by being included within the $h$-interval. Figures $7 \mathrm{e}$ and $7 \mathrm{f}$ show that the $h$-intervals generated by the proposed VS-FR-SM and VS-FR-SM are zero, when $x_{1}$ is at zero. Hence, the fuzziness of the attractiveness is zero given that $x_{1}$ is zero, as the $h$-intervals are zero. Figures $7 \mathrm{a}, 7 \mathrm{~b}, 7 \mathrm{c}$ and $7 \mathrm{~d}$ also show the $h$-intervals generated by the existing methods, TS-FR, TS-SR-FR, FC-FR and LW-FR, when $x_{1}$ is at zero. These figures show that the fuzzy intervals range from 3 to 4.5 , given that $x_{1}$ is zero. Therefore, more fuzziness is generated by the existing methods compared with the proposed methods, when $x_{1}$ is at zero. Then, we consider $x_{1}$ is at 1 . Figures $7 \mathrm{a}, 7 \mathrm{~b}, 7 \mathrm{c}$, $7 \mathrm{~d}, 7 \mathrm{e}$ and $7 \mathrm{f}$ show that similar $h$-intervals are generated based on the proposed and the existing methods, where the $h$-intervals are around 2 to 4 . As smaller fuzzy intervals are generated by the proposed methods when $x_{1}$ is at zero and similar fuzzy intervals are generated when $x_{1}$ is at one, the overall fuzziness generated by the proposed methods is smaller compared with the existing methods. Therefore, unnecessary fuzziness is less likely to be estimated by the proposed methods, while the developed $h$-intervals cover all the collected data.

Similarly, Figures $8 \mathrm{a}$ to $8 \mathrm{f}$ illustrate the models which correlate the attractiveness with $x_{4}$. They show similar characteristics in that smallest $h$-intervals are generated by the proposed VS-FR-SM and VS-FR-SM, compared with the existing methods. Therefore, the proposed VS-FR-SM and VS-FR-GA outperform the other tested methods.

Tables 5, 6 and 7 show the three fuzzy criteria, AFS, IC and MFC, obtained by all the methods. These results demonstrate the effectiveness of the proposed methods compared to the existing methods. Table 5 shows the AFSs which illustrate the overall fuzzy spreads of a model covering the samples. It also shows the ranks with respect to the AFSs for all the tested methods. It shows that the proposed VS-FR-SM and VS-FR-GA, can generally generate smaller AFSs, compared with the four existing methods, TS-FR, FC-FR, LW-FR and TS-SR-FR. The ranks for the proposed methods are higher than those for the existing methods. Improvement can be obtained by the proposed VS-FR-SM. Hence, unnecessary fuzziness is less likely to be generated by the proposed methods than by the existing methods.

Table 6 shows the IC for the six tested methods. When the IC value is larger, the estimates generated by the fuzzy regression model are better fitted to the collected data. Table 6 shows that the proposed methods generally obtain higher ICs compared with the existing methods, while FC-FR is the poorest method of all. The proposed methods obtain higher ranks in terms of IC. Therefore, the proposed methods are able to generate a better fit than the other tested methods. Among the five tested methods, the FC-FR generates the most unfitted models for the collected data. Table 7 shows the MFC for the six tested methods. When the MFC is larger, the fuzzy regression models can generate better memberships for the collected data; moreover, the overall fuzziness is smaller. Table 7 shows that the proposed methods, VS-FR-SM and VS-FR-GA, and the existing method, FC-FR, can achieve better MFC compared with the other three existing methods, TS-FR, LW-FR and TS-SR-FR. The poorest method is TS-SR-FR, although it performs well in terms of IC. As FC-FR is developed by optimizing the MFC of the fuzzy regression models, the MFC achieved by the FC-FR is generally good. However, the models generated by FC-FR perform poorest in terms of IC. It is a trade-off when using FC-FR. These results show that the proposed methods, VS-FR-SM and VS-FR-GA, generally outperform the other existing methods in terms of the three fuzzy criteria, AFS, IC and MFC.

The better results can be explained by the thrid-order polynomial functions that the proposed methods use to represent the fuzzy spreads while the other existing methods only use the linear polynomial function. Therefore, smaller fuzzy intervals can be generated by the proposed methods in order to cover all the samples; also, the fuzzy intervals are a better fit to the samples. Better AFS, IC and MFC can generally be achieved by the proposed methods. To show the superior of the proposed method, we consider the relative improvements between the fuzz criteria achieved by the proposed methods to that achieved by the existing method. The relative improvement is formulated in (17). It is similar to the variation measure which indicates performance difference between two models [32].

$$
\text { Relative }=\frac{\left(\begin{array}{l}
\text { Fuzzy criteria by } \\
\text { proposed method }
\end{array}\right)-\left(\begin{array}{l}
\text { Fuzzy criteria by } \\
\text { existing method }
\end{array}\right)}{\left(\begin{array}{l}
\text { Fuzzy criteria by } \\
\text { existing method }
\end{array}\right)}
$$


Table 4 Fuzzy regression models for affective design of electric irons

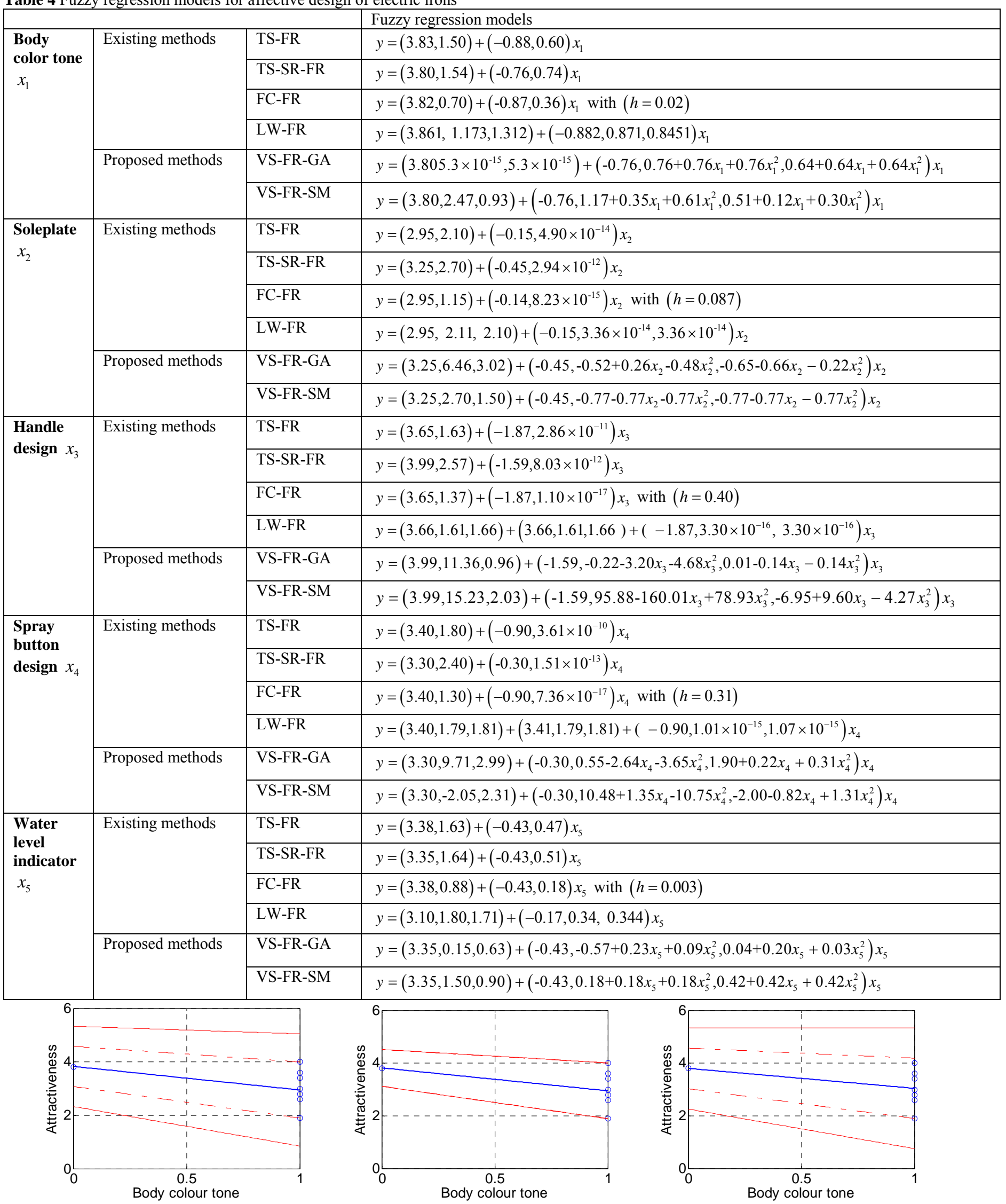


$x_{1}$ to attractiveness $y$

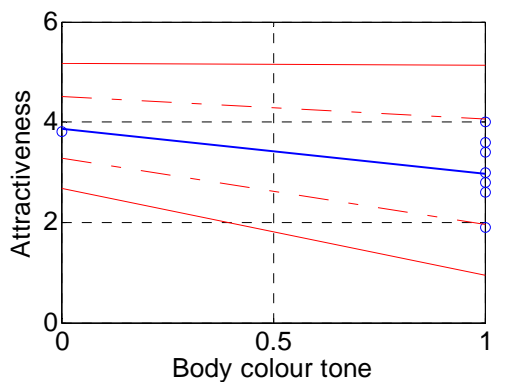

Figure 7d LW-FR correlates body colour tone $x_{1}$ to attractiveness $y$

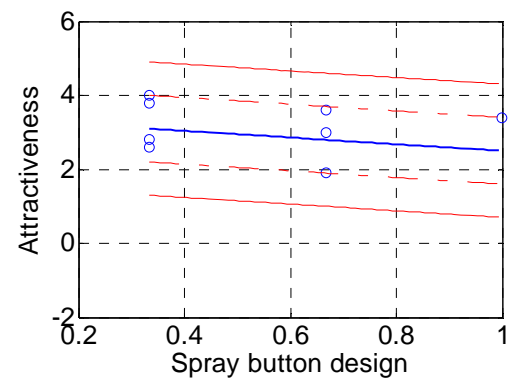

Figure 8a TS-FR correlates spray button design $x_{4}$ to attractiveness $y$

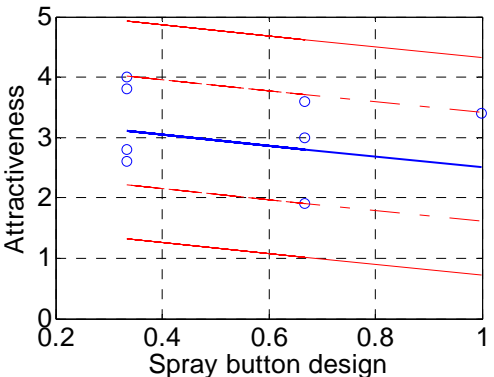

Figure 8d VS-FR-GA correlates spray button design $x_{4}$ to attractiveness $y$

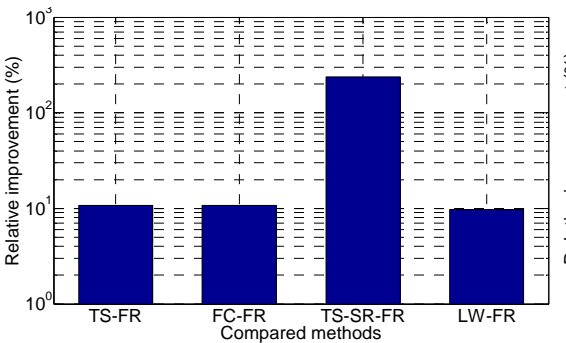

Figure 9a Rel. improvements for AFS (iron) $x_{1}$ to attractiveness $y$

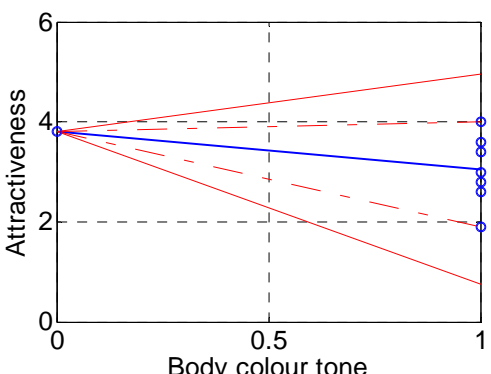

Figure 7e VS-FR-GA correlates body colour tone $x_{1}$ to attractiveness $y$

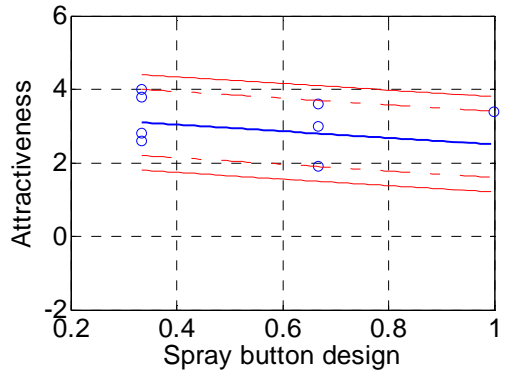

Figure 8b FC-FR correlates spray button design $x_{4}$ to attractiveness $y$

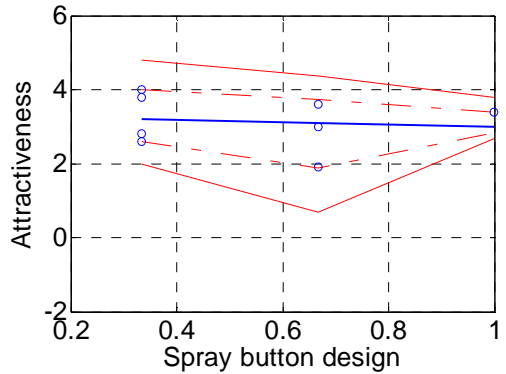

Figure 8e VS-FR-GA correlates spray button design $x_{4}$ to attractiveness $y$

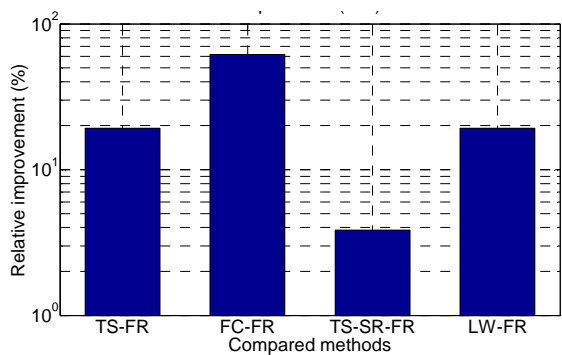

Figure 9b Rel. improvements for IC (iron) tone $x_{1}$ to attractiveness $y$

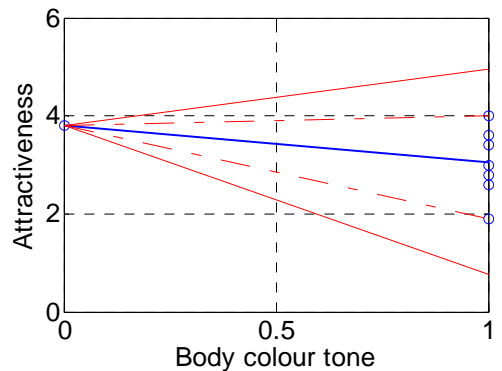

Figure 7f VS-FR-SM correlates body colour tone $x_{1}$ to attractiveness $y$

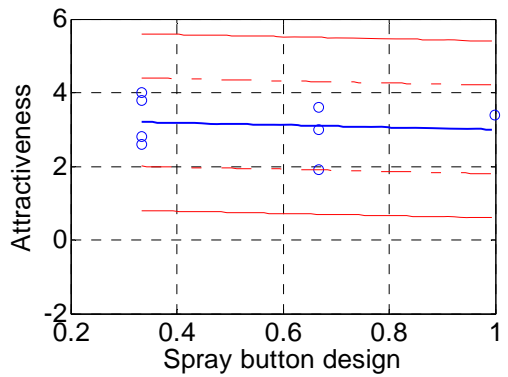

Figure 8c TS-SR-FR correlates spray button design $x_{4}$ to attractiveness $y$

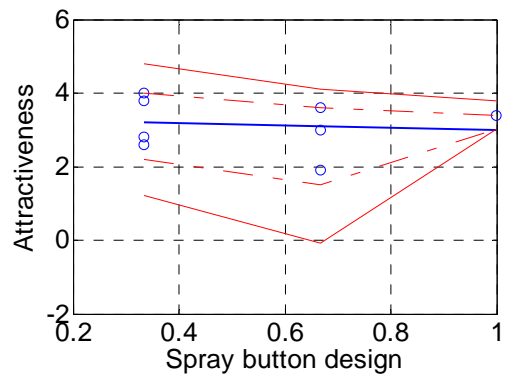

Figure 8f VS-FR-SM correlates spray button design $x_{4}$ to attractiveness $y$

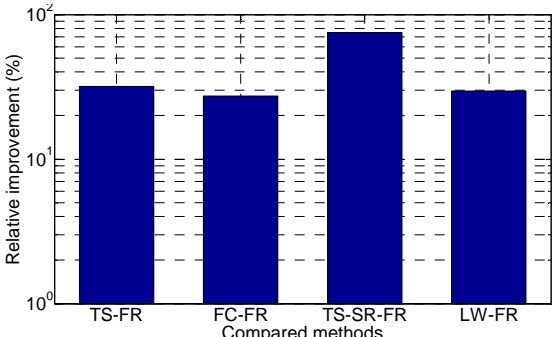

Figure 9c Rel. improvements for MFC (iron)

Table 5 Average fuzzy spread (AFS) for the electric iron design (*The ranks of each method are bracketed)

\begin{tabular}{|l|c|c|c|c|c|c|c|c|}
\hline & Model & $\begin{array}{c}\text { Body } \\
\text { color tone } \\
x_{1}\end{array}$ & Soleplate $x_{2}$ & $\begin{array}{c}\text { Handle } \\
\text { design } x_{3}\end{array}$ & $\begin{array}{c}\text { Spray } \\
\text { button } \\
\text { design } x_{4}\end{array}$ & $\begin{array}{c}\text { Water level } \\
\text { indicator } x_{5}\end{array}$ & $\begin{array}{c}\text { All features, } x_{1}, x_{2}, \\
x_{3}, x_{4} \text { and } x_{5}\end{array}$ & $\begin{array}{c}\text { Ave. } \\
\text { rank }\end{array}$ \\
\hline \multirow{2}{*}{$\begin{array}{l}\text { Existing } \\
\text { methods }\end{array}$} & TS-FR & $2.03(5)$ & $2.14(5)$ & $1.68(5)$ & $1.83(4)$ & $1.86(3)$ & $1.03(5)$ \\
& FC-FR & $2.01(4)$ & $2.13(4)$ & $1.63(4)$ & $1.87(5)$ & $1.92(4)$ & $1.03(4)$ \\
\cline { 2 - 9 } & TS-SR-FR & $3.14(6)$ & $3.14(6)$ & $3.16(6)$ & $3.14(6)$ & $3.14(6)$ & 4.140 \\
\cline { 2 - 9 } & LW-FR & $1.93(3)$ & $2.11(3)$ & $1.61(3)$ & $1.79(3)$ & $1.97(5)$ & $1.14(6)$ & 6.00 \\
\hline \multirow{2}{*}{$\begin{array}{l}\text { Proposed } \\
\text { methods }\end{array}$} & VS-FR-SM & $1.84(1)$ & $1.68(1)$ & $1.17(2)$ & $1.46(1)$ & $1.65(2)$ & $0.93(3)$ & 3.33 \\
\cline { 2 - 9 } & VS-FR-GA & $1.88(2)$ & $1.69(2)$ & $1.14(1)$ & $1.48(2)$ & $1.63(1)$ & $0.94(2)$ & 1.33 \\
\hline
\end{tabular}


Table 6 Index of confidence (IC) for the electric iron design (*The ranks of each method are bracketed)

\begin{tabular}{|l|c|c|c|c|c|c|c|c|}
\hline & Model & $\begin{array}{c}\text { Body } \\
\text { color tone } \\
x_{1}\end{array}$ & Soleplate $x_{2}$ & $\begin{array}{c}\text { Handle } \\
\text { design } x_{3}\end{array}$ & $\begin{array}{c}\text { Spray } \\
\text { button } \\
\text { design } x_{4}\end{array}$ & $\begin{array}{c}\text { Water level } \\
\text { indicator } x_{5}\end{array}$ & $\begin{array}{c}\text { All features, } x_{1}, x_{2}, \\
x_{3}, x_{4} \text { and } x_{5}\end{array}$ & $\begin{array}{c}\text { Ave. } \\
\text { rank }\end{array}$ \\
\hline \multirow{2}{*}{$\begin{array}{l}\text { Existing } \\
\text { methods }\end{array}$} & TS-FR & $0.23(4)$ & $0.23(5)$ & $0.21(4)$ & $0.22(5)$ & $0.23(5)$ & $0.21(4)$ \\
& FC-FR & $0.00(6)$ & $0.01(6)$ & $0.13(6)$ & $0.07(6)$ & $0.00(6)$ & $0.10(6)$ \\
\cline { 2 - 9 } & TS-SR-FR & $0.24(3)$ & $0.24(3)$ & $0.25(5)$ & $0.24(1)$ & $0.24(2)$ & $0.25(2)$ & 2.67 \\
\cline { 2 - 9 } & LW-FR & $0.23(5)$ & $0.23(4)$ & $0.21(3)$ & $0.22(4)$ & $0.23(4)$ & $0.21(5)$ & 4.17 \\
\hline \multirow{2}{*}{$\begin{array}{l}\text { Proposed } \\
\text { methods }\end{array}$} & VS-FR-SM & $0.27(2)$ & $0.34(1)$ & $0.44(2)$ & $0.23(3)$ & $0.26(1)$ & $0.26(1)$ \\
\cline { 2 - 8 } & VS-FR-GA & $0.29(1)$ & $0.34(2)$ & $0.44(1)$ & $0.23(2)$ & $0.24(3)$ & $0.25(3)$ & 2.00 \\
\hline
\end{tabular}

Table 7 Mean fuzzy credibility (MFC) for the electric iron design (*The ranks of each method are bracketed.)

\begin{tabular}{|c|c|c|c|c|c|c|c|c|}
\hline & Model & $\begin{array}{c}\text { Body } \\
\text { color tone } \\
x_{1}\end{array}$ & Soleplate $x_{2}$ & $\begin{array}{c}\text { Handle } \\
\text { design } x_{3}\end{array}$ & $\begin{array}{c}\text { Spray } \\
\text { button } \\
\text { design } x_{4}\end{array}$ & $\begin{array}{l}\text { Water level } \\
\text { indicator } x_{5}\end{array}$ & $\begin{array}{c}\text { All features, } x_{1}, x_{2} \text {, } \\
x_{3}, x_{4} \text { and } x_{5}\end{array}$ & $\begin{array}{l}\text { Ave. } \\
\text { rank }\end{array}$ \\
\hline \multirow{4}{*}{$\begin{array}{l}\text { Existing } \\
\text { methods }\end{array}$} & TS-FR & $0.20(5)$ & $0.17(4)$ & $0.18(5)$ & $0.18(2)$ & $0.21(4)$ & $0.30(5)$ & 4.167 \\
\hline & FC-FR & $0.29(1)$ & $0.22(3)$ & $0.18(3)$ & $0.19(1)$ & $0.27(1)$ & $0.32(3)$ & 2.000 \\
\hline & TS-SR-FR & $0.08(6)$ & $0.02(6)$ & $0.08(6)$ & $0.05(6)$ & $0.10(6)$ & $0.11(6)$ & 6.000 \\
\hline & LW-FR & $0.21(4)$ & $0.17(5)$ & $0.18(4)$ & $0.18(3)$ & $0.19(5)$ & $0.31(4)$ & 4.167 \\
\hline \multirow{2}{*}{$\begin{array}{l}\text { Proposed } \\
\text { methods }\end{array}$} & VS-FR-SM & $0.26(3)$ & $0.25(1)$ & $0.25(1)$ & $0.14(4)$ & $0.21(3)$ & $0.44(1)$ & 2.167 \\
\hline & VS-FR-GA & $0.25(2)$ & $0.25(2)$ & $0.24(2)$ & $0.15(5)$ & $0.21(2)$ & $0.43(2)$ & 2.500 \\
\hline
\end{tabular}

In (17), the fuzzy criteria can either be AFS, IC or MFC. The model is better when either MFC or IC is large. The model is better when the AFS is smaller. Therefore (17) can directly be used when either MFC or IC is considered. The plus sign needs to be changed to minus in (17) when AFS is considered. Figures $9 \mathrm{a}, 9 \mathrm{~b}$ and $9 \mathrm{c}$ show the relative improvements for AFS, IC and MFC respectively. Due to the page limit, we consider the models with all affective features. The relative improvements between the proposed VS-FR-SM and the existing methods are considered The figures show that generally more than $10 \%$ improvements can be achieved by the proposed VS-FR-SM for AFS, IC and MFC. These improvements demonstrate the superior of the proposed method.

To demonstrate how the fuzzy model can be used for product development, we consider the fuzzy regression model, $\tilde{F}_{V S}(\bar{x})=\left(f_{V S}^{C}(\bar{x}), f_{V S}^{L}(\bar{x}), f_{\text {Lin }}^{R}(\bar{x})\right)$, developed by VS-FR-GA, where $\bar{x}=\left(x_{1}, x_{2}, x_{3}, x_{4}, x_{5}\right)$ are all objective features. We consider $\tilde{F}_{V S}(\bar{x})$, which is better than the other models developed by the existing methods in terms of AFS, IC and MFC. Based on $\tilde{F}_{V S}(\bar{x})$, the fuzzy optimization is given in (18) which maximizes the affective quality $f_{V S}^{C}(\bar{x})$ constrained with a predefined uncertainty limit, $\lambda^{*}$, in quality estimation [27].

$\max f_{v S}^{C}(\bar{x})=4.7+0.3 x_{1}+0.6 x_{2}-2.4 x_{3}-0.8 x_{4}-0.4 x_{5} ;$; (18) subject to $\left(f_{V S}^{L}(\bar{x})+f_{L i n}^{R}(\bar{x})\right) \leq \lambda^{*}$, where

$$
\begin{aligned}
& f_{V S}^{L}(\bar{x})=3.94+\left(1-0.01 x_{1}+0.75 x_{1}^{2}\right) x_{1}+\left(-0.44+0.6 x_{2}+0.8 x_{2}^{2}\right) x_{2} \\
& \quad+\left(-0.2+0.18 x_{3}+0.1 x_{3}^{2}\right) x_{3}+\left(0.03-2.33 x_{4}+0.53 x_{4}^{2}\right) x_{4} \\
& \quad+\left(0.37-0.54 x_{5}+1.06 x_{5}^{2}\right) x_{5} \text {, and } \\
& \quad f_{V S}^{L}(\bar{x})=0.37+\left(-0.6-0.2 x_{1}+1.1 x_{1}^{2}\right) x_{1}+\left(0.2+1.0 x_{2}+0.7 x_{2}^{2}\right) x_{2} \\
& \quad+\left(0.24-0.75 x_{3}-0.28 x_{3}^{2}\right) x_{3}+\left(0.04-1.03 x_{4}-0.04 x_{4}^{2}\right) x_{4} \\
& \quad+\left(0.15+0.35 x_{5}+0.17 x_{5}^{2}\right) \cdot x_{5}
\end{aligned}
$$

By solving (18), the exact solution for the five objective features can be determined. Based on the exact solution, an electric iron can be designed with the maximum affective quality constrained with the required uncertainty. In new product development, some objective features are required to be predefined and cannot be changed. Despite the exact solution, inexact solutions can be determined by solving (18), when some of the objective features are predefined by the product designers For example, we consider that the body color tone and the soleplate are required to be predefined with warm tone (i.e. $x_{1}=0.5$ ) and round tip (i.e. $x_{1}=0.5$ ) respectively. By solving (18) which substituting $x_{1}=0.5$ and $x_{1}=0.5$, we can determine the inexact solutions for $x_{3}, x_{4}$ and $x_{5}$ which represent handle, spray button and water level indicator for the electric iron respectively. Detailed discussion of determination of inexact solutions can be referred to [50].

\section{B. Image quality assessment Modelling}

Subjective image quality assessment (IQA) is typically performed to evaluate the affective quality of an image, which is important for many image processing applications such as image/video compression and image reconstruction [6]. In subjective IQA, a group of participants typically score the affective qualities of images and then the opinion scores are combined to produce Mean Opinion Scores (MOS), and the MOS can indicate the performance of image processing systems. Since MOS are obtained by subjective experiments, they cannot be obtained and deployed in real time. Therefore, it is essential to develop IQA models that predict the MOS [6]. These models typically take either an image analysis approach or a human visual system approach [51]. Both approaches, but more predominantly the former approach, use statistical regression [6] to estimate the MOS from objective features of an image. In our earlier work, we proposed fuzzy regression to develop IQA models in order to address the fuzziness of the MOS [22]. To further improve the reliability of IQA, the proposed fuzzy regression with varying spreads is used here and evaluated in the following subsections.

1) Data description

The effectiveness of IQA models based on the proposed fuzzy regression is evaluated based on the image quality database of Ponomarenko et al. [28]. This database contains 1725 distorted images which were developed based on 25 reference images 
contaminated with 17 types of distortion and 4 levels for each type of distortion. Including the 25 reference images, 1725 image samples are considered. Table 8 in the appendix illustrates the five image subsets namely Noise3, JPEG, Exotic, Actual and Full, which are involved with five different sets of distortion types [28]. In Table 8, the distortion types that belong to a given image subset are marked by ' + '; also the number of distorted images in each image subset is shown. As an illustration in the appendix, Figures 10a to 10e, and Figures 11a to $11 \mathrm{e}$ show two image samples that are distorted by Additive Gaussian noise and Spatially-correlated noise with 4 distortion levels respectively. Seventeen objective features, $x_{1}$ to $x_{17}$, have been used to evaluate the distorted images. Details on the 17 objective features can be referred to [28]. Table 8 shows the most significant objective features to each image subset. The distorted images were evaluated with the MOS by performing 256428 subjective IQAs, where the assessments involved interviewees from 5 countries (Finland, France, Italy, Ukraine, and USA). Based on the MOS, five IQA models can be developed, each of which corresponds to an image subset, in order to correlate MOS and the objective features.

2) Numerical results

Table 9 shows the fuzzy regression models developed by the 3 existing methods, TS-FR, FC-FR and TS-SR-FR, and the proposed method, VS-FR-GA. These fuzzy regression models correlate the MOS with the most significant objective feature of the image subset. Based on these IQA models, the MOS and its fuzziness can be predicted. As a large amount of MOS data is used in the IQA model development, the integer programming problem formulated by (13a) to (13e) has numerous constraints. To develop the fuzzy regression models, we need to solve this integer programming problem while simultaneously addressing all the constraints. Only infeasible solutions can be generated by the VS-FR-SM involving a simplex method which is a local search approach, and feasible solutions can be found by the VS-FR-GA takes the global search approach using the GA-solver. Hence, the results of VS-FR-GA are reported.

Due to the constraints of limited space, we illustrate the models developed for only two image subsets, Noise 3 and JPEG, where the models are correlated with the most significant objective features, PSNRHVS $x_{8}$ and PSNRHVSM $x_{11}$ respectively. Figures $12 \mathrm{a}$ to $12 \mathrm{e}$ illustrate the fuzzy regression models that correlate $x_{8}$ to MOS, where the image subset, Noise3, is used. Figures 12a to 12e show the IQA models generated by existing methods TS-FR, TS-SR-FR, FC-FR, LW-FR and VS-FR-GA respectively. They show that the overall $h$-intervals generated by the proposed VS-FR-GA are smaller than those generated by existing methods. Hence, the overall fuzziness generated by the proposed method is smaller compared with that of the existing methods. Therefore, unnecessary fuzziness is less likely to be generated by the proposed method used for the MOS predictions. Similar results can be found in Figures 13a to 13e which illustrate the models corresponding with $x_{11}$ for the image subset, JPEG. Smaller overall fuzziness is generated by the proposed VS-FR-GA, thereby further demonstrating that the proposed VS-FR-GA outperforms the other tested methods.
Tables 10, 11 and 12 show the three fuzzy criteria, AFS, IC and MFC, for the IQA models corresponding to the most significant objective feature respectively. These results attempt to demonstrate the effectiveness of the proposed method in developing the IQA models when compared with the existing methods. Table 10 shows the AFSs which evaluate the overall fuzzy spreads of the IQA models developed by the tested methods. It also shows the ranks with respect to the AFSs achieved by the IQA models. It shows that the proposed VS-FR-GA can generate smaller AFSs than do the existing methods, TS-FR, FC-FR, TS-SR-FR and LW-FR. The ranks for the proposed methods are the highest. Therefore, the VS-FR-GA can develop better models which are less likely to generate unnecessary fuzziness. Table 11 shows the IC obtained by the tested methods. It shows that the VS-FR-GA can generally obtain higher ICs compared with the existing methods, while FC-FR is the poorest method as the smallest IC is obtained. The proposed VS-FR-GA obtains the highest rank for IC, and therefore it can generate a better fit for IQA models to the MOS data than the existing methods. However, the FC-FR generates the most unfitted models to the MOS data. Table 12 shows the MFC obtained by the tested methods. It shows that the proposed method, and the existing FC-FR can obtain better MFC compared with TS-FR, TS-SR-FR and LW-FR. However, the FC-FR can generate the poorest models with the lowest IC. It is the trade-off of using FC-FR. Therefore, these results show the proposed method generally outperforms the other existing methods in terms of the three fuzzy criteria.

Tables 13 to 15 show the three fuzzy criteria achieved by the IQA models which correlate the MOS with all the 17 objective features, $x_{1}$ to $x_{17}$. They demonstrate the effectiveness of the proposed VS-FR-GA when developing the IQA models that involve more independent regressors. Tables 13, 14 and 15 respectively show that better AFS, IC and MFC can be achieved by the VS-FR-GA than those achieved by the existing methods. These results show that the proposed VS-FR-GA can generally produce better IQA models with more regressors. When small or large numbers of regressors are used for developing the IQA models, the proposed VS-FR-GA generally outperforms the existing methods. As VS-FR-GA uses the third-order polynomial functions to represent the fuzzy spreads, smaller fuzzy intervals are generated to cover all the MOS data. Hence, better AFS, IC and MFC can generally be achieved by the proposed method compared with the other tested methods which use only linear polynomials to represent the fuzzy intervals. The relative improvement in (17) is used to determine the superior of the proposed VS-FR-GA comparing with the other existing methods. Due to the page limit, we consider the models developed by two databases, ACTUAL and FULL. Figures 14 and 15 show the relative improvements for ACTUAL and FULL respectively. Figures $14 \mathrm{a}$ to $\mathrm{c}$ show the relative improvement for the three fuzzy criteria AFS, IC and MFC respectively. They show that generally more than $10 \%$ improvements can be achieved by the proposed method compared with the existing methods. Similar relative improvements with $10 \%$ can be found in Figures $15 \mathrm{a}$ to $\mathrm{c}$ for FULL database. These relative improvements demonstrate the superior of the proposed method. 
Table 9 Fuzzy regression models for image quality assessment

\begin{tabular}{|c|c|c|c|}
\hline & & & Fuzzy regression models \\
\hline \multirow{5}{*}{$\begin{array}{c}\text { Noise3 to } \\
\text { PSNRHVS } \\
x_{8}\end{array}$} & \multirow{4}{*}{$\begin{array}{l}\text { Existing } \\
\text { methods }\end{array}$} & TS-FR & $y=(-2.13,0.73)+(0.28,0.21) x_{8}$ \\
\hline & & TS-SR-FR & $y=(-0.87,0.07)+(0.17,0.14) x_{8}$ \\
\hline & & FC-FR & $y=(-2.13,0.73)+(0.26,0.20) x_{8}$ with $(h=0.021)$ \\
\hline & & LW-FR & $y=(1.67,3.89,3.88)+\left(4.93,2.54 \times 10^{-17}, 2.57 \times 10^{-17}\right) x_{8}$ \\
\hline & Proposed & VS-FR-GA & $y=(-0.87,0.34,-0.5)+\left(0.17,0.15-0.6 \times 10^{-3} x_{8}-0.7 \times 10^{-5} x_{8}^{2}, 0.13-0.5 \times 10^{-3} x_{8}-0.5 \times 10^{-5} x_{8}^{2}\right) x_{8}$ \\
\hline \multirow{5}{*}{$\begin{array}{c}\text { JPEG to } \\
\text { PSNRHVSM } \\
x_{11}\end{array}$} & \multirow{4}{*}{$\begin{array}{l}\text { Existing } \\
\text { methods }\end{array}$} & TS-FR & $y=(-1.76,0.24)+(0.18,0.09) x_{11}$ \\
\hline & & TS-SR-FR & $y=(-0.93,0.40)+(0.15,0.12) x_{11}$ \\
\hline & & FC-FR & $y=\left(-1.76,1.00 \times 10^{-13}\right)+(0.18,0.04) x_{11}$ with $(h=0.087)$ \\
\hline & & LW-FR & $y=(-0.78,3.08,2.08)+(0.14,-0.00,0.02) x_{11}$ \\
\hline & Proposed & VS-FR-GA & $y=(-0.9,1.0,1.9)+\left(0.15,5+9.7 \times 10^{-6} x_{11}+1.6 \times 10^{-7} x_{11}^{2}, 0.02+4.1 \times 10^{-6} x_{11}+5.7 \times 10^{-7} x_{11}^{2}\right) x_{11}$ \\
\hline \multirow{5}{*}{$\begin{array}{l}\text { Exotic to } \\
\text { MSSIM } x_{2}\end{array}$} & \multirow{4}{*}{$\begin{array}{l}\text { Existing } \\
\text { methods }\end{array}$} & TS-FR & $y=(0.26,4.33)+\left(5.19,8.03 \times 10^{-12}\right) x_{2}$ \\
\hline & & TS-SR-FR & $y=(-1.51,7.87)+\left(6.82,3.81 \times 10^{-10}\right) x_{2}$ \\
\hline & & FC-FR & $y=(0.26,2.19)+\left(5.19,2.81 \times 10^{-16}\right) x_{2}$ with $(h=0.40)$ \\
\hline & & LW-FR & $y=(0.26,4.32,4.34)+\left(4.93,1.04 \times 10^{-9}, 1.04 \times 10^{-9}\right) x_{2}$ \\
\hline & Proposed & VS-FR-GA & $y=(-1.51,2.23,0.64)+\left(6.82,-0.31+0.04 x_{2}+0.18 x_{2}^{2},-0.32+3.42 x_{2}+4.13 x_{2}^{2}\right) x_{2}$ \\
\hline \multirow{5}{*}{$\begin{array}{c}\text { Actual to } \\
\text { PSNRHVSM } \\
x_{11}\end{array}$} & \multirow[t]{4}{*}{ Tested methods } & TS-FR & $y=\left(2.11,2.82 \times 10^{-16}\right)+(5.11,0.24) x_{11}$ \\
\hline & & TS-SR-FR & $y=\left(1.72,4.64 \times 10^{-15}\right)+(5.60,0.260) x_{11}$ \\
\hline & & FC-FR & $y=\left(2.12,2.80 \times 10^{-16}\right)+(5.11,0.13) x_{11}$ with $(h=0.31)$ \\
\hline & & LW-FR & $y=(2.11,4.42,4.41)+\left(5.11,6.93 \times 10^{-19}, 6.93 \times 10^{-19}\right) x_{11}$ \\
\hline & Proposed & VS-FR-GA & $y=(1.72,-2.99,2.77)+\left(5.60,0.17-0.38 x_{11}+5.45 x_{11}^{2}, 0.60+0.62 x_{11}-1.02 x_{11}^{2}\right) x_{11}$ \\
\hline \multirow{5}{*}{$\begin{array}{c}\text { Fill to } \\
\text { MSSIM } x_{2}\end{array}$} & \multirow{4}{*}{$\begin{array}{l}\text { Existing } \\
\text { methods }\end{array}$} & TS-FR & $y=(-0.17,5.19)+\left(5.19,1.38 \times 10^{-16}\right) x_{2}$ \\
\hline & & TS-SR-FR & $y=(-2.59,10.02)+\left(7.86,1.20 \times 10^{-9}\right) x_{2}$ \\
\hline & & FC-FR & $y=(-0.17,2.73)+\left(5.19,8.13 \times 10^{-14}\right) x_{2}$ with $(h=0.003)$ \\
\hline & & LW-FR & $y=(-0.17,5.18,5.20)+\left(5.19,2.32 \times 10^{-13}, 2.36 \times 10^{-13}\right) x_{2}$ \\
\hline & Proposed & VS-FR-GA & $y=(-2.59,2.63,-3.63)+\left(7.86,0.57-0.25 x_{2}-0.43 x_{2}^{2}, 0.07+3.58 x_{2}-9.99 x_{2}^{2}\right) x_{2}$ \\
\hline
\end{tabular}

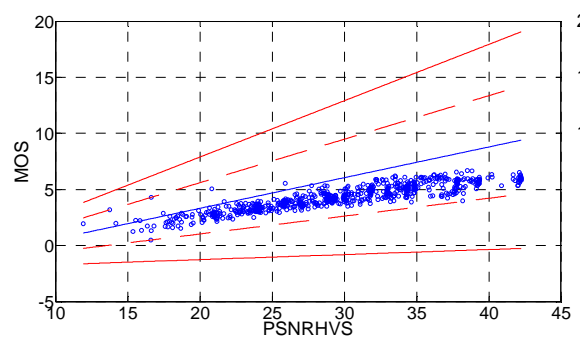

Fig. 12a TS-FR correlates PSNRHVS $x_{8}$ to MOS $y$ under the data subset (Noise3)

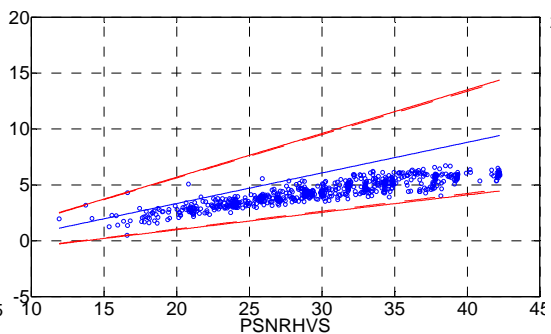

Fig. 12b FC-FR correlates PSNRHVS $x_{8}$ to MOS $y$ under the data subset (Noise3)

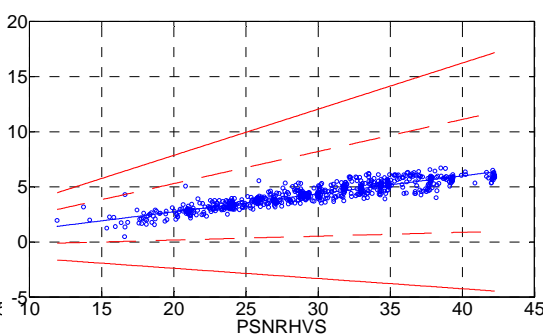

Fig. 12c TS-SR-FR correlates PSNRHVS $x_{8}$ to MOS $y$ under the data subset (Noise3) 


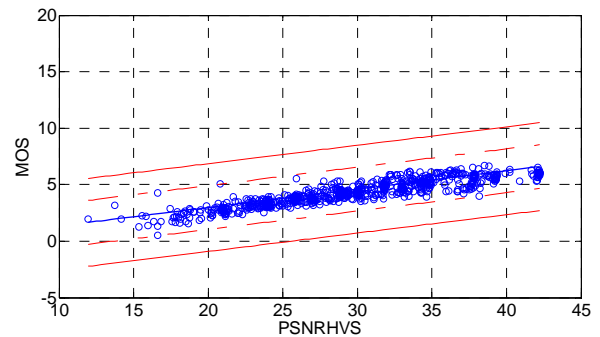

Fig. 12d LW-FR correlates PSNRHVS $x_{8}$ to MOS $y$ under the data subset (Noise3)

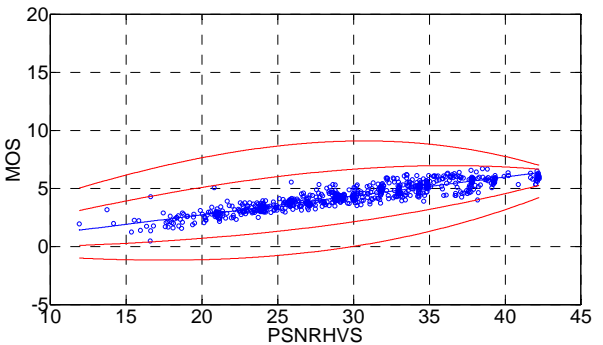

Fig. 12e VS-FR-GA correlates PSNRHVS $x_{8}$ to MOS $y$ under the data subset (Noise3)

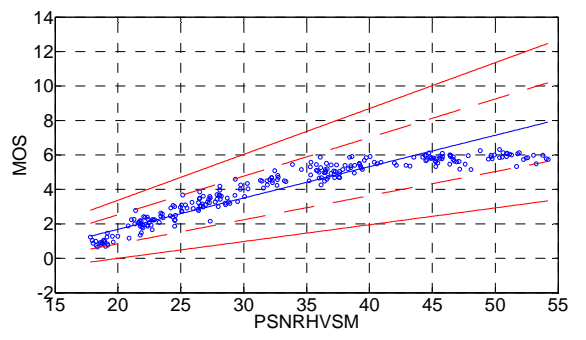

Fig. 13a TS-FR correlates PSNRHVSM $x_{11}$ to MOS $y$ under the data subset (JPEG)

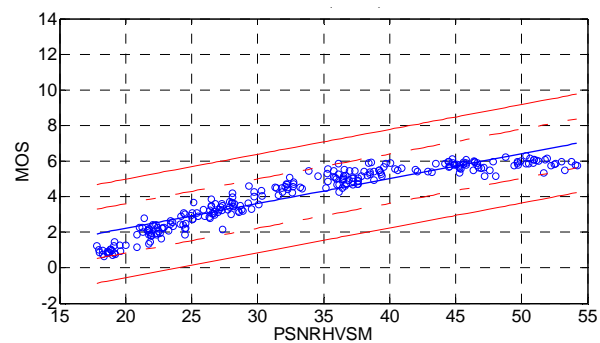

Fig. 13d LW-FR correlates PSNRHVSM $x_{11}$ to MOS $y$ under the data subset (JPEG)

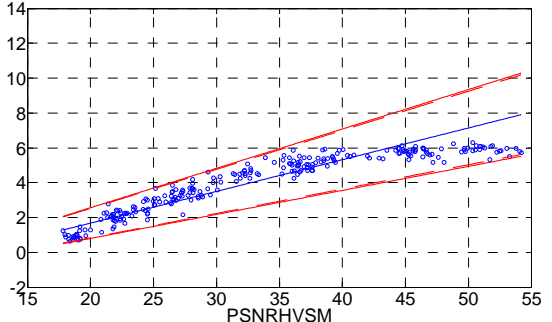

Fig. 13b FC-FR correlates PSNRHVSM $x_{11}$ to MOS $y$ under the data subset (JPEG)

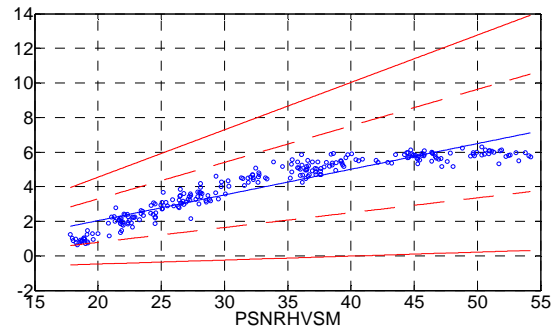

Fig. 13c TS-SR-FR correlates PSNRHVSM $x_{11}$ to MOS $y$ under the data subset (JPEG)

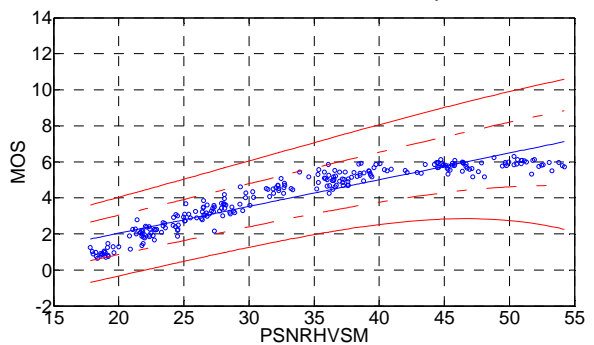

Fig. 13e VS-FR-GA correlates PSNRHVSM $x_{11}$ to MOS $y$ under the data subset (JPEG)

Table 10 Average fuzzy spread (AFS) for the IQA models correlated to the most significant objective feature

\begin{tabular}{|l|c|c|c|c|c|c|c|}
\hline & Model & $\begin{array}{c}\text { PSNRHVS } x_{8} \\
\text { (Noise3) }\end{array}$ & $\begin{array}{c}\text { PSNRHVSM } \\
x_{11} \text { (JPEG) }\end{array}$ & $\begin{array}{c}\text { MSSIM } x_{2} \\
\text { (Exotic) }\end{array}$ & $\begin{array}{c}\text { PSNRHVSM } x_{11} \\
\text { (Actual) }\end{array}$ & $\begin{array}{c}\text { MSSIM } x_{2} \\
\text { (Full) }\end{array}$ & $\begin{array}{c}\text { Ave. } \\
\text { rank }\end{array}$ \\
\hline \multirow{5}{*}{ Existing methods } & TS-FR & $3.40(2)$ & $1.42(3)$ & $2.17(2)$ & $3.75(3)$ & $2.60(2)$ & 2.40 \\
& FC-FR & $3.50(3)$ & $1.48(4)$ & $2.19(3)$ & $3.97(4)$ & $5.62(5)$ & 3.60 \\
\cline { 2 - 8 } & TS-SR-FR & $3.80(4)$ & $2.13(5)$ & $3.94(5)$ & $4.17(5)$ & $5.01(4)$ & 4.60 \\
\cline { 2 - 8 } & LW-FR & $3.89(5)$ & $1.39(2)$ & $2.16(1)$ & $3.71(2)$ & $2.60(3)$ & 2.60 \\
\hline Proposed methods & VS-FR-GA & $1.19(1)$ & $1.33(1)$ & $2.22(4)$ & $2.23(1)$ & $2.17(1)$ & 1.60 \\
\hline
\end{tabular}

*The ranks of each method are bracketed.

Table 11 Index of confidence (IC) for the IQA models correlated to the most significant objective feature

\begin{tabular}{|l|c|c|c|c|c|c|c|}
\hline & Model & $\begin{array}{c}\text { PSNRHVS } x_{8} \\
\text { (Noise3) }\end{array}$ & $\begin{array}{c}\text { PSNRHVSM } \\
x_{11} \text { (JPEG) }\end{array}$ & $\begin{array}{c}\text { MSSIM } x_{2} \\
\text { (Exotic) }\end{array}$ & $\begin{array}{c}\text { PSNRHVSM } x_{11} \\
\text { (Actual) }\end{array}$ & $\begin{array}{c}\text { MSSIM } x_{2} \\
\text { (Full) }\end{array}$ & $\begin{array}{c}\text { Ave. } \\
\text { rank }\end{array}$ \\
\hline \multirow{3}{*}{ Existing methods } & TS-FR & $0.23(4)$ & $0.24(4)$ & $0.24(3)$ & $0.25(3)$ & $0.24(3)$ & 3.40 \\
& FC-FR & $0.20(5)$ & $0.21(4)$ & $0.21(5)$ & $0.24(5)$ & $0.23(5)$ & 4.80 \\
\cline { 2 - 8 } & TS-SR-FR & $0.25(2)$ & $0.25(2)$ & $0.25(2)$ & $0.25(2)$ & $0.25(2)$ & 2.00 \\
\cline { 2 - 8 } & LW-FR & $0.25(3)$ & $0.24(3)$ & $0.24(4)$ & $0.24(4)$ & $0.24(4)$ & 3.60 \\
\hline Proposed methods & VS-FR-GA & $0.33(1)$ & $0.28(1)$ & $0.32(1)$ & $0.36(1)$ & $0.36(1)$ & 1.00 \\
\hline
\end{tabular}

*The ranks of each method are bracketed.

Table 12 Mean fuzzy credibility (MFC) for the IQA models correlated to the most significant objective feature

\begin{tabular}{|l|c|c|c|c|c|c|c|}
\hline & Model & $\begin{array}{c}\text { PSNRHVS } x_{8} \\
\text { (Noise3) }\end{array}$ & $\begin{array}{c}\text { PSNRHVSM } \\
x_{11} \text { (JPEG) }\end{array}$ & $\begin{array}{c}\text { MSSIM } x_{2} \\
\text { (Exotic) }\end{array}$ & $\begin{array}{c}\text { PSNRHVSM } x_{11} \\
\text { (Actual) }\end{array}$ & $\begin{array}{c}\text { MSSIM } x_{2} \\
\text { (Full) }\end{array}$ & $\begin{array}{c}\text { Ave. } \\
\text { rank }\end{array}$ \\
\hline \multirow{5}{*}{ Existing methods } & TS-FR & $0.06(5)$ & $0.16(3)$ & $0.09(3)$ & $0.03(4)$ & $0.06(3)$ & 3.60 \\
& FC-FR & $0.08(3)$ & $0.24(2)$ & $0.15(1)$ & $0.11(1)$ & $0.14(1)$ & 1.60 \\
\cline { 2 - 8 } & TS-SR-FR & $0.06(4)$ & $0.02(5)$ & $0.05(5)$ & $0.04(3)$ & $0.04(4)$ & 4.20 \\
\cline { 2 - 8 } & LW-FR & $0.12(2)$ & $0.15(4)$ & $0.09(4)$ & $0.03(5)$ & $0.06(5)$ & 4.00 \\
\hline Proposed methods & VS-FR-GA & $0.43(1)$ & $0.26(1)$ & $0.09(2)$ & $0.04(2)$ & $0.07(2)$ & 1.60 \\
\hline
\end{tabular}

*The ranks of each method are bracketed. 
Table 13 AFS for the IQA models correlated to all objective features (*The ranks of each method are bracketed)

\begin{tabular}{|l|c|c|c|c|c|c|c|}
\hline & Model & Noise3 & JPEG & Exotic & Actual & Full & Ave. rank \\
\hline Existing methods & TS-FR & $2.09(2)$ & $1.19(3)$ & $2.95(3)$ & $2.66(3)$ & $3.12(3)$ & 2.80 \\
\cline { 2 - 8 } & FC-FR & $3.82(5)$ & $1.14(2)$ & $3.64(5)$ & $1.33(2)$ & $1.56(2)$ & 3.20 \\
\cline { 2 - 8 } & TS-SR-FR & $3.04(4)$ & $1.91(5)$ & $1.47(2)$ & $3.82(5)$ & $4.23(5)$ & 4.20 \\
\cline { 2 - 8 } & LW-FR & $2.09(1)$ & $1.36(4)$ & $2.95(4)$ & $2.66(4)$ & $3.12(4)$ & 3.40 \\
\hline Proposed method & VS-FR-GA & $2.56(3)$ & $0.95(1)$ & $0.61(1)$ & $0.61(1)$ & $0.95(1)$ & 1.40 \\
\hline
\end{tabular}

Table 14 IC for the IQA models correlated to all objective features (*The ranks of each method are bracketed)

\begin{tabular}{|l|c|c|c|c|c|c|c|}
\hline & Model & Noise3 & JPEG & Exotic & Actual & Full & Ave. rank \\
\hline Existing methods & TS-FR & $0.24(3)$ & $0.24(3)$ & $0.24(3)$ & $0.24(3)$ & $0.23(3)$ & 3.00 \\
\cline { 2 - 8 } & FC-FR & $0.25(2)$ & $0.21(5)$ & $0.24(2)$ & $0.21(5)$ & $0.21(5)$ & 3.20 \\
\cline { 2 - 8 } & TS-SR-FR & $0.21(5)$ & $0.25(2)$ & $0.20(5)$ & $0.25(2)$ & $0.27(2)$ & 3.20 \\
\cline { 2 - 8 } & LW-FR & $0.24(4)$ & $0.24(4)$ & $0.24(4)$ & $0.24(4)$ & $0.23(4)$ & 4.00 \\
\hline Proposed method & VS-FR-GA & $0.49(1)$ & $0.42(1)$ & $0.30(1)$ & $0.30(1)$ & $0.29(1)$ & 1.00 \\
\hline
\end{tabular}

Table 15 MFC for the IQA models correlated to all objective features (*The ranks of each method are bracketed.)

\begin{tabular}{|l|c|c|c|c|c|c|c|}
\hline & Model & Noise3 & JPEG & Exotic & Actual & Full & Ave. rank \\
\hline Existing methods & TS-FR & $0.20(3)$ & $0.30(3)$ & $0.14(3)$ & $0.16(3)$ & $0.14(3)$ & 3.00 \\
\cline { 2 - 9 } & FC-FR & $0.31(2)$ & $0.43(2)$ & $0.20(2)$ & $0.25(2)$ & $0.22(2)$ & 2.00 \\
\cline { 2 - 9 } & TS-SR-FR & $0.12(5)$ & $0.24(5)$ & $0.12(5)$ & $0.12(5)$ & $0.11(5)$ & 5.00 \\
\cline { 2 - 9 } & LW-FR & $0.20(4)$ & $0.29(4)$ & $0.14(4)$ & $0.16(4)$ & $0.14(4)$ & 4.00 \\
\hline Proposed method & VS-FR-GA & $0.41(1)$ & $0.60(1)$ & $0.39(1)$ & $0.37(1)$ & $0.23(1)$ & 1.00 \\
\hline
\end{tabular}

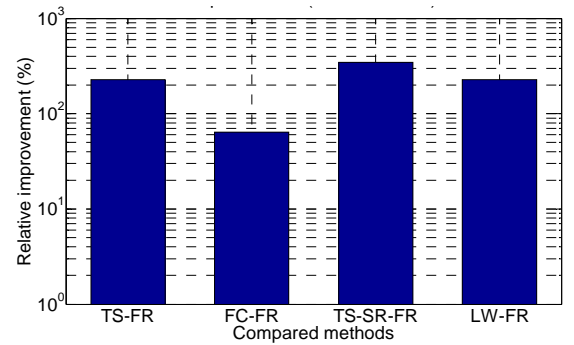

Figure 14a Improve. AFS (ACTUAL)

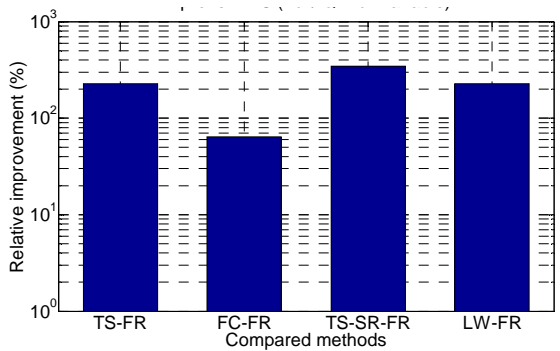

Figure 15a Improve. AFS (FULL)

\section{C.Discussion}

The fuzzy regression models can be used to predict the affective qualities and uncertainty on evaluating the qualities. For both electric iron design and IQA, we consider the fuzzy regression models developed by the VS-FR-GA which are better than the existing methods in terms of AFS, IC and MFC. Equation (19) shows the model for the electric iron design, when we consider different spray buttons which are either flat $\left(x_{4}=0.33\right)$, curvy $\left(x_{4}=0.67\right)$ or handle-shape $\left(x_{4}=1\right)$.

$$
\begin{aligned}
y= & \left(-0.30,0.55-2.64 x_{4}-3.65 x_{4}^{2}, 1.90+0.22 x_{4}+0.31 x_{4}^{2}\right) x_{4}+ \\
& (3.30,9.71,2.99)
\end{aligned}
$$

Similar to the analysis of fuzzy sets in $[52,53]$, the patterns of fuzzy estimates are analyzed based on (19). The $x$-axis of Figures $16 \mathrm{a}$ to $16 \mathrm{c}$ shows the fuzzy estimates of attractiveness $(y)$, when $x_{4}=0.33, x_{4}=0.67$ and $x_{4}=1$ are substituted into

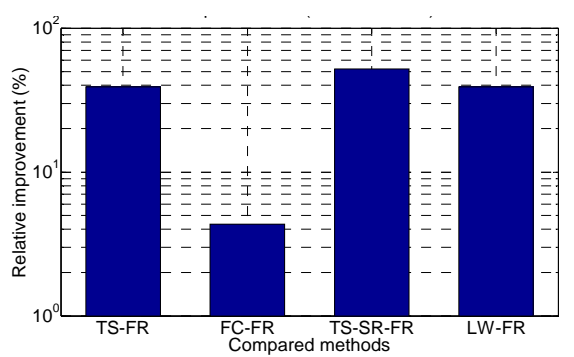

Figure 14c Improve. MFC (ACTUAL)

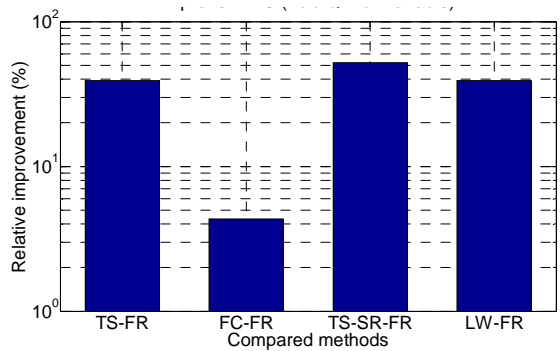

Figure 15c Improve. MFC (FULL) (20) respectively. When the patterns of fuzzy estimates are given, the memberships with respect to $y$ can be indicated. The figures show the samples covered by the fuzzy estimates and they also show the memberships of the samples. The memberships of the samples are higher than 0.5 , as the model is developed with the membership constrained with $h=0.5$. Affective quality estimated for flat spray button is higher than those of the curvy and handle-shape. However, uncertainty estimated for the curvy is larger than the flat which is larger than the handle-shape, as the base of the membership triangular of curvy is larger than that of flat which is larger than that of handle-shape. When an electric iron is designed, both estimated quality and estimated uncertainty need to be considered. Although higher attractiveness can be achieved with the flat spray button, higher uncertainty exists on it. A tradeoff between affective quality and uncertainty needs to be determined. 


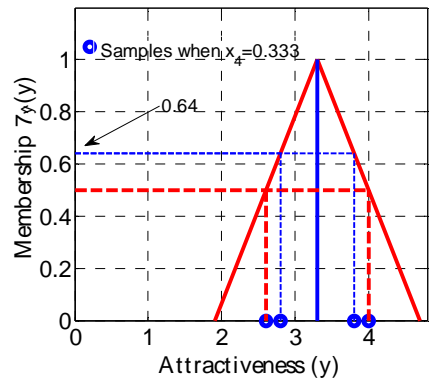

Fig. 16a Spray button with flat $\left(x_{4}=0.33\right)$

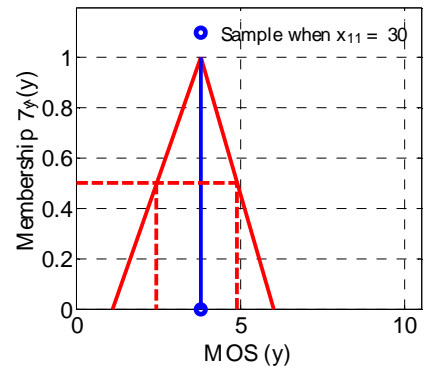

Fig. 17a PSNRHVSM $\left(x_{11}=30\right)$

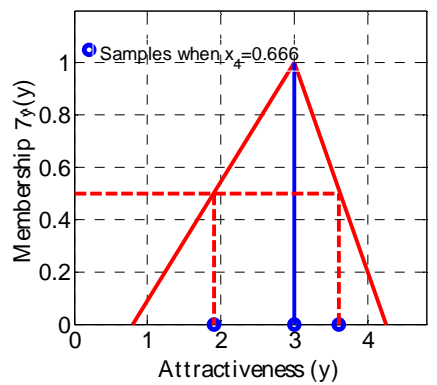

Fig. 16b Spray button with curvy $\left(x_{4}=0.67\right)$

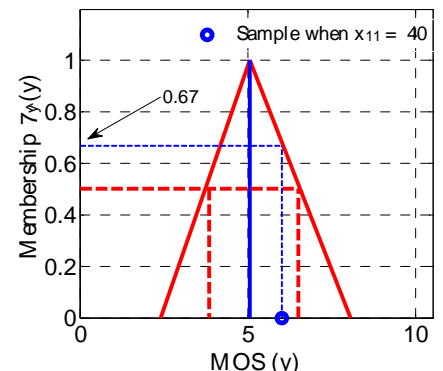

Fig. 17b PSNRHVSM $\left(x_{11}=40\right)$

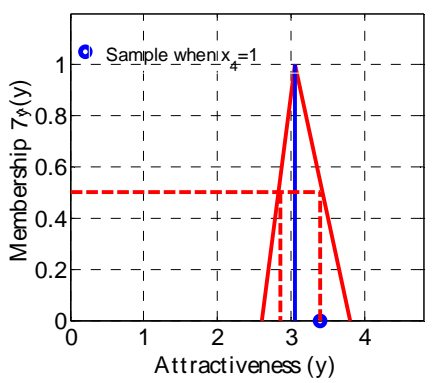

Fig. 16c Spray button with handle-shape $\left(x_{4}=1\right)$

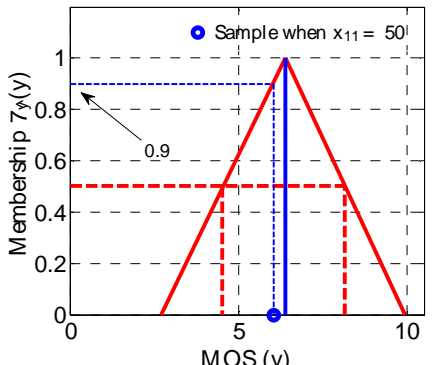

Fig. 17c PSNRHVSM $\left(x_{11}=50\right)$
For the IQA, we consider the models developed for estimating affective qualities of images which are contaminated by JPEG. The following model (20) developed by VS-FR-GA is considered, as the model is better than those developed by the existing methods.

$$
\begin{gathered}
y=(-0.93,1.02,1.86)+\left(0.15,0.05+1 \times 10^{-5} x_{11}+2 \times 10^{-4} x_{11}^{2},\right. \\
\left.0.02+4 \times 10^{-5} x_{11}+5.66 x_{11}^{2}\right) x_{11}
\end{gathered}
$$

The $x$-axis of Figures 17 a to $c$ show the fuzzy estimates of the MOS when the PSNRHVSM with $x_{11}=30, x_{11}=40$ and $x_{11}=50$ are measured on the distorted images respectively. We can see that the memberships of the samples are higher than 0.5 , where the membership of the sample with $x_{11}=30$ is 1 ; the one with $x_{11}=40$ is 0.67 ; and the one with $x_{11}=50$ is 0.9 . The center of the membership triangular shows estimate of the image quality and the base shows the uncertainty of the quality. The figures indicate that the estimated quantities and uncertainties of the MOS increase when the measured PSNRHVSM increases from 30 to 50 . When the PSNRHVSM of an image is given, the model can be used to predict the quantity of MOS and the uncertainty on evaluating the MOS.

The proposed approaches, VS-FR-SM and VS-FR-GA, are based on the optimization methods, simplex method and genetic algorithm respectively. Generally, the computational time required by genetic algorithms are higher than those of the local search method such as simplex method, as more computational evaluations are generally performed in genetic algorithms. Hence, VS-FR-GA requires more computational time than those required by the VS-FR-SM. For the electric iron design with one independent variable, VS-FR-GA required 24.5 seconds for determining the feasible solutions and VS-FR-SM requires 0.02 seconds. For the IQA with one independent variable, VS-FR-GA required 4.4 minutes in average for determining the feasible solutions but VS-FR-SM cannot find the feasible solutions. Also VS-FR-GA can find the feasible solutions for IQA with full independent variables while VS-FR-SM cannot. The computational times required by
VS-FR-GA are reasonable, as affective quality models are developed offline and real time implementation is not required. However, VS-FR-SM engaged with simplex method can only develop feasible solutions for the electric iron design which consists of 8 samples. The VS-FR-SM cannot develop feasible solutions for IQA which consists of more than 225 samples. Although smaller computational time is required by VS-FR-SM, VS-FR-SM cannot generate a feasible affective model when the number of samples is large. Therefore, we can only use VS-FR-SM when the number of samples is small. When the number of samples is large, VS-FR-GA is used.

\section{V.CONCLUSION}

In this paper, a novel fuzzy regression method has been proposed to take into account the fuzziness generated by the affective quality assessments, which cannot be done with the commonly-used statistical regression method. The proposed approach involves varying spreads in order to take into account the fuzziness in affective qualities. It overcomes the existing fuzzy regression methods that tackle only a single characteristic where the amount of fuzziness is linearly correlated with the independent and dependent variables. In fact, the fuzziness of dependent variables may decrease or remain unchanged when increasing the values of independent variables particularly when assessing affective quality. Therefore, unnecessary fuzziness is more likely to be generated by the existing fuzzy regression methods when this assumption is not valid. The proposed fuzzy regression uses a varying spread based on a third-order polynomial, in order to take into account the nonlinear and nonsymmetrical fuzziness caused by affective quality assessment.

The effectiveness of the proposed varying spread fuzzy regression is evaluated using two case studies, the affective design of an electric iron and image quality assessment, both of which are fuzzy. They attempt to evaluate the effectiveness of the proposed method in predicting different types of affective qualities, where discrete and continuous data, large and small amount of data, and data with varying fuzziness are considered. The proposed approach is compared with the state-of-art fuzzy 
regression approaches, the recently developed fuzzy regression approach and the fuzzy regression approach particularly developed for overcoming the problem of increasing fuzziness. Results indicate that better fuzzy regression models can be generated by the proposed approach in terms of three fuzzy criteria namely, index of confidence, mean fuzzy credibility and average fuzzy spread of each estimate. The better results can be explained by the third-order polynomial functions that the proposed methods use in order to represent the fuzzy spreads, while the other existing methods use only the linear polynomial function. These results also show that the proposed fuzzy regression can simulate increased, decreased or unchanged variances of the affective qualities which are observed as differences between objective features. Therefore, the smaller fuzzy intervals can be generated by the proposed method in order to cover all the observed data; moreover, the fuzzy intervals are a better fit to the data. Hence, the proposed method can obtain better results in terms of the three fuzzy criteria.

In the future, we will further improve the effectiveness of the proposed method. Here, the proposed method models the central of fuzzy data as a linear relation. In the future, we will improve the strategy of modeling the centrals of fuzzy data. The nonlinearity of fuzzy data will be taken into account, which would increase the fitting capability of the model.

Acknowledgement: The first author would like to thank C.K. Kwong for many useful discussions and providing the data sets of the electric iron design.

\section{REFERENCES}

[1] J. A. Russell, "Core affect and the psychological construction of emotion," Psychological Review, vol. 110, no. 1, pp. 145-172, 2003.

[2] P. Zhang, and N. Li, "The importance of Affective Quality," Communications of the ACM, vol. 48, no. 9, pp. 105-108, 2005.

[3] M. Kurosu, and K. Kashimura, "Apparent usability vs. inherent usability: experimental analysis on the determinants of the apparent usability," in Proceedings of the Conference Companion on Human Factors in Computing Systems, 1995, pp. 292-293.

[4] D. A. Norman, Emotion and Attractive, Interactions, p.^pp. 36 42, 2002

[5] M. Seckler, K. Opwis, and A. N. Tuch, "Linking objective design factors with subjective aesthetics: an experimental study on how structure and color of websites affect the facets of users' visual aesthetic perception," Computers in Human Behavior, vol. 49, pp. 375-389, 2015

[6] U. Engelke, A. Maeder, and H. J. Zepernick, "Human observer confidence in image quality assessment," Signal Processing: Image Communication, vol. 27, pp. 935-947, 2012.

[7] D. G. Kleinbaum, and L. L. Kupper, Applied regression analysis and other multivariable methods: Duxbury Press, 1978.

[8] W. H. Mormann, B. Stawarczyk, A. Ender, B. Sener, T. Attin, and A. Mehl, "Wear characteristics of current aesthetic dental restorative $\mathrm{CAD} / \mathrm{CAM}$ materials: two-body wear, gloss retention, roughness and Martens hardness," Journal of Mechanical Behavior Biomedical Materials, vol. 20, pp. 113-125, 2013.

[9] J. Georgii, M. Eder, K. Burger, S. Klotz, F. Ferstl, L. Kovacs, and R. Westermann, "A computational tool for preoperative breast augmentation planning in aesthetic plastic surgery," IEEE Journal of Biomedical and Health Informatics, vol. 18, no. 3, pp. 907-919, 2014.

[10] M. Zhang, D. Wang, Z. Wang, X. Zhang, G. Zhang, and Y. Xu, "Assessing the aesthetic value of urban forests and associated individual differences: an example from the Loess Plateau," Advanced Science and Technology Letters, vol. 49, pp. 274-280, 2014.
[11] L. Marchesotti, N. Murray, and F. Peronnin, "Discovering beautiful attributes for aesthetic image analysis," International Journal of Computer Vision, vol. 113, pp. 246-266, 2015.

[12] M. T. Tsai, K. A. Wang, Y. Liu, and J. S. Hong, "Perceived visual aesthetics of text-overlaid images: computational models and experimental research for white-space fraction," International Journal of Human Computer Interaction, vol. 29, pp. 1-23, 2014.

[13] J. Tarvainen, M. Sjoberg, S. Westman, J. Laaksonen, and P. Oittinen, "Content-based prediction of movie style, aesthetics, and affect: data set and baseline experiments," IEEE Transactions on Multimedia, vol. 16, no. 8, pp. 2085-2098, 2014.

[14] C. Barnes, and S. P. Lillford, "Decision support for the design of affective products," Journal of Engineering Design, vol. 20, no. 5, pp. 477-492, 2009.

[15] K. Choi, and C. Jun, "A systematic approach to the Kansei factors of tactile sense regarding the surface roughness," Applied Ergonomics, vol. 38, no. 1, pp. 53-63, 2007.

[16] M. Nagamachi, "Perspectives and the new trend of Kansei/affective engineering," The TQM Journal, vol. 20, no. 4, pp. 290-298, 2008.

[17] G. Elkharraz, S. Thumfart, D. Akay, C. Eitzinger, and B. Henson, "Making tactile textures with predefined affective properties," IEEE Transactions on Affective Computing, vol. 5, no. 1, pp. 57-70, 2014.

[18] H. Tanaka, S. Uejima, and K. Asai, "Linear regression analysis with fuzzy model," IEEE Transactions on Systems, Man and Cybernetics, vol. 12, pp. 903-907, 1982.

[19] B. Hesehmaty, and A. Kandel, "Fuzzy linear regression and its applications to forecasting in uncertain environment," Fuzzy Sets and Systems, vol. 15, pp. 159-191, 1985.

[20] Y. Shimizu, and Y. Jindo, "A fuzzy logic analysis method for evaluation human sensitivities," International Journal of Industrial Ergonomics, vol. 15, pp. 39-47, 1995.

[21] K. Y. Chan, C. K. Kwong, T. S. Dillon, and K. Y. Fung, "An intelligent fuzzy regression approach for affective product design that captures nonlinearity and fuzziness," Journal of Engineering Design, vol. 22, no. 8, pp. 523-542, 2011.

[22] K. Y. Chan, and U. Engelke, "Fuzzy regression for perceptual image quality assessment," Engineering Applications of Artificial Intelligence, vol. 43, pp. 102-110, 2015.

[23] C. Kao, and C. L. Chyu, "Least squares estimates in fuzzy regression analysis," European Journal of Operational Research, vol. 148, pp. 426-435, 2003.

[24] P. D. Urso, "Linear regression analysis for fuzzy/crisp input and fuzzy/crisp output data," Computational Statistics and Data Analysis, vol. 42, no. 1-2, pp. 47-72, 2003.

[25] M.M.Nasrabadi, and E.Nasrabadi, "A mathematical-programming approach to fuzzy linear regression analysis," Applied Mathematics and Computation, vol. 155, no. 3, pp. 873-881, 2004.

[26] J. Lu, and R. Wang, "An enhanced fuzzy linear regression model with more flexible spreads," Fuzzy Sets and Systems, vol. 160, pp. 2505-2523, 2009.

[27] C. K. Kwong, H. Jiang, and X. G. Luo, "AI-based methodology of integrating affective design, engineering, and marketing for defining design specifications of new products," Engineering Applications of Artificial Intelligence, vol. 1, 2016.

[28] N. Ponomarenko, L. Jin, O. Ieremeiev, V. Lukin, K. Egiazarian, J. Astola, B. Vozel, K. Chehdi, M. Carli, F. Battisti, and C.-C. J. Kuo, "Image database TID2013: Peculiarities, results and perspectives," Signal Processing: Image Communication, vol. 30, no. 30, pp. 57-77, 2015.

[29] D. A. Savic, and W. Pedrycz, "Evaluation of fuzzy linear regression models," Fuzzy Sets and Systems, vol. 39, no. 1, pp. $51-63,1991$.

[30] X. Liu, and Y. Chen, "A systematic approach to optimizing $h$ value for fuzzy linear regression with symmetric triangular fuzzy numbers," Mathematical Problems in Engineering, 2013. 
[31] Y. Liu, Y. Chen, J. Zhou, and S. Zhong, "Fuzzy linear regression models for QFD using optimized h values," Engineering Applications of Artificial Intelligence, vol. 39, pp. 45-54, 2015.

[32] H. F. Wang, and R. C. Tsaur, "Insight of fuzzy regression model," Fuzzy Sets and Systems, vol. 112, pp. 355-369, 2000.

[33] R. Likert, "A technique for the measurement of attitudes," Archives of Psychology, vol. 22, no. 140, pp. 55, 1932.

[34] L. A. Zadeh, "The concept of a Linguistic variable and its application to approximate reasoning - I," Information Sciences, vol 8, pp. 199-249, 1975.

[35] Q. H. Thu, M. N. Garcia, F. Speranza, P. Corriveau, and A. Raake, "Study of rating scales for subjective assessment of high-definition video," IEEE Transactions on Broadcasting, vol. 57, no. 1, pp. 1-14, 2011

[36] H. M. Jiang, C. K. Kwong, W. H. Ip, and Z. Q. Chen, "Chaos-based fuzzy regression approach to modelling customer satisfaction for product design," IEEE Transactions on Fuzzy Systems, vol. 21, no. 5, pp. 926-936, 2013.

[37] P. Nanda, J. Bos, K. L. Kramer, C. Hay, and J. Ignacz, "Effect of smartphone aesthetic design on users' emotional reaction," The TQM Journal, vol. 20, no. 4, pp. 348-355, 2008.

[38] D. Dubois, and H. Prade, "Gradualness, uncertainty and bipolarity: Making sense of fuzzy sets," Fuzzy Sets and Systems, vol. 192, no. 1 , pp. $3-24,2012$.

[39] Y. H. Chang, "Hybrid fuzzy least squares regression analysis and its reliability measures," Fuzzy Sets and Systems, vol. 119, pp. 225-246, 2001

[40] K. J. Kim, H. Moskowitz, and M. Koksalan, "Fuzzy versus statistical linear regression," European Journal of Operational Research, vol. 92, pp. 417-434, 1996.

[41] D. G. Bails, and L. C. Peppers, Chapter 6: Multiple Regression, Business Fluctuations: Forecasting Techniques and Applications, New York: Prentice-Hall, 1982

[42] S. Muzzioli, A. Ruggieri, and B. D. Baets, "A comparison of fuzzy methods for the estimation of the implied volatility smile function," Fuzzy Sets and Systems, vol. 226, pp. 131-143, 2015.

[43] H. C. Chen, J. H. Fang, S. M. E. Kortright, and D. S. Chen, "Novel approaches to the determination of archie parameters II: fuzzy regression analysis," SPE: Advanced Technology Series, vol. 3, no. 1, pp. 44-52, 1995
[44] J. H. Kim, and H. Myung, "Evolutionary programming techniques for constrained optimization problems," IEEE Transactions on Evolutionary Computation, vol. 1, no. 2, pp. 129-140, 1997.

[45] A. V. Mogilenko, D. A. Pavlyuchenko, and V. Z. Manusov, "Development of fuzzy regression models using genetic algorithms," International Journal of Uncertainty, Fuzziness and Knowledge-Based Systems, vol. 11, no. 4, pp. 429-444, 2003.

[46] J. Schaffer, R. Caruana, L. Eshelman, and R. Das, "A study of control parameters affecting online performance of genetic algorithms for function optimization," in Third International Conference on Genetic Algorithms, 1989, pp. 51-61.

[47] C. K. Kwong, Y. Chen, K. Y. Chan, and X. Luo, "A generalised fuzzy least-squares regression approach to modelling relationships in QFD," Journal of Engineering Design, vol. 21, no. 5, pp. 601-613, 2010.

[48] Y. Liu, J. Zhou, and Y. Chen, "Using fuzzy nonlinear regression to identify the degree of compensation among customer requirements in QED," Neurocomputing, vol. 142, pp. 115-124, 2014.

[49] Y. Chen, J. Tang, R. K. Fung, and Z. Ren, "Fuzzy regression based mathematical programming model for quality function deployment," International Journal of Product Research, vol. 42, no. 5, pp. 1009-1027, 2004

[50] H. Bai, and C. K. Kwong, "Inexact genetic algorithm approach to target values setting of engineering requirements in QFD," International Journal of Production Research, vol. 41, no. 16, pp. 3861-3881, 2003.

[51] J. Wu, W. Lin, G. Shi, and A. Liu, "Reduced-reference image quality assessment with visual information fidelity," IEEE Transactions on Multimedia, vol. 15, no. 7, pp. 1700-1705, 2013.

[52] J. V. d. Oliveira, "Semantic constraints for membership function optimization," IEEE Transactions on Systems, Man and Cybernetic. Part A: Systems and Humans, vol. 29, no. 1, pp. 128-138, 1999.

[53] P. Pulkkinen, and H. Koivisto, "A Dynamically Constrained Multiobjective Genetic Fuzzy System for Regression Problems," IEEE Transactions on Fuzzy Systems, vol. 18, no. 1, pp. 161 - 177, 2010 .

\section{Appendix}

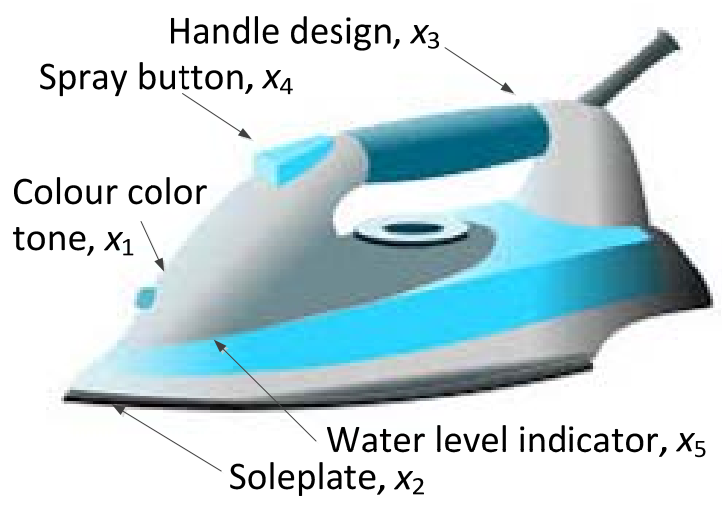

Figure 6 The five objective features of electric irons. 
Table 2 Morphological description of electric irons [27]

\begin{tabular}{|l|l|l|}
\hline Objective features & (1) Warm tone & Category \\
\hline Body colour tone $x_{1}$ & & (2) Cold tone \\
\hline Soleplate $x_{2}$ & (1) Sharp tip &
\end{tabular}

Table 3 Categorical data of electric irons [27]

\begin{tabular}{|c|c|c|c|c|c|c|c|c|c|}
\hline Item & Category and qualitative value & A & B & C & D & $\mathbf{E}$ & $\mathbf{F}$ & G & $\mathbf{H}$ \\
\hline 2. Soleplate $x_{2}$ & $\begin{array}{l}\text { (1) Sharp tip } \\
\text { (2) Round tip } \\
\text { Oualitative value }\end{array}$ & 0.50 & $\begin{array}{c}X \\
100\end{array}$ & $\begin{array}{c}X \\
100\end{array}$ & 0.50 & 0.50 & 0.50 & 0.50 & 0.50 \\
\hline 3. Handle design $x_{3}$ & $\begin{array}{l}\text { (1) Embedded type } \\
\text { (2) "" type } \\
\text { (3) “" " type } \\
\text { (4) "T" type } \\
\text { Qualitative value }\end{array}$ & 0.25 & 0.75 & $\begin{array}{c}X \\
1.00\end{array}$ & 0.50 & $\mathrm{X}$ & 075 & $\mathrm{X}$ & 0.50 \\
\hline 4. Spray button design $x_{4}$ & $\begin{array}{l}\text { (1) Flat } \\
\text { (2) Curvy } \\
\text { (3) Handle-shape } \\
\text { Qualitative value }\end{array}$ & 0.33 & 0.66 & 0.33 & 0.66 & 0.33 & 0.33 & 0.66 & $\begin{array}{c}\mathrm{X} \\
1.00\end{array}$ \\
\hline 5. Water level indicator $x_{5}$ & $\begin{array}{l}\text { (1) Transparent } \\
\text { (2) Sandblasted } \\
\text { Qualitative value }\end{array}$ & 0.50 & $\begin{array}{c}\mathrm{X} \\
1.00\end{array}$ & 0.50 & $\begin{array}{c}\mathrm{X} \\
1.00\end{array}$ & $\begin{array}{c}\mathrm{X} \\
1.00\end{array}$ & $\begin{array}{c}\mathrm{X} \\
1.00\end{array}$ & 0.50 & 0.50 \\
\hline
\end{tabular}

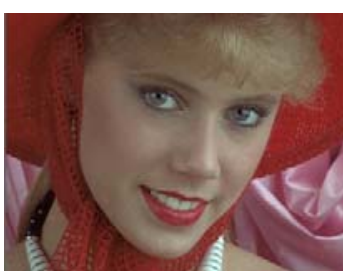

Fig. 10a Original image

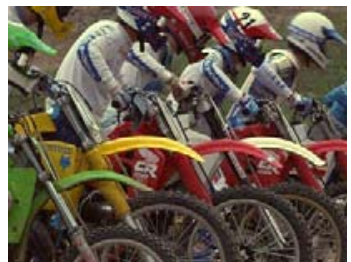

Fig. 11a Original image

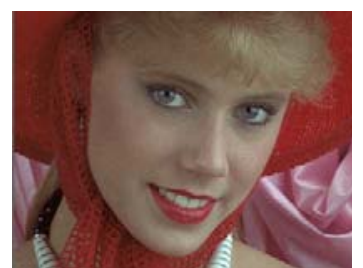

Fig. 10b Additive Gaussian noise in level 1

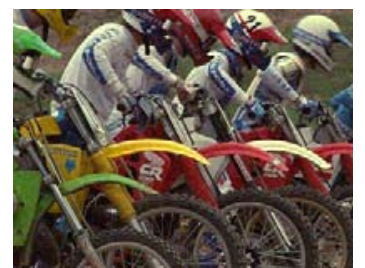

Fig. 11b Spatially correlated noise in level 1

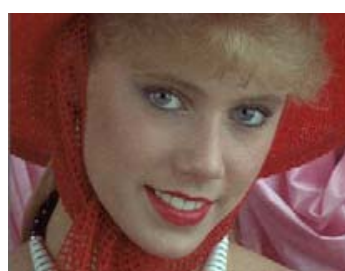

Fig. 10c Additive Gaussian noise in level 2

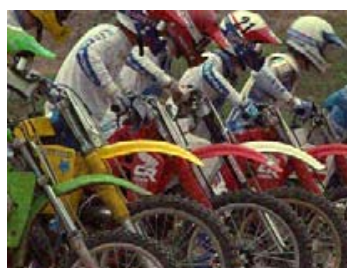

Fig. 11c Spatially correlated noise in level 2

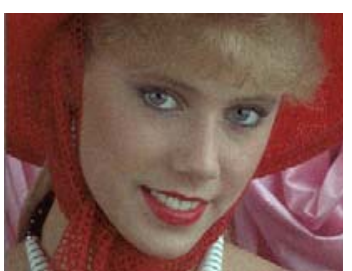

Fig. 10d Additive Gaussian noise in level 3

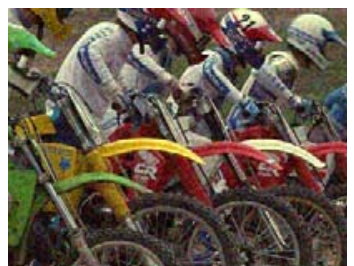

Fig. 11d Spatially correlated noise in level 3

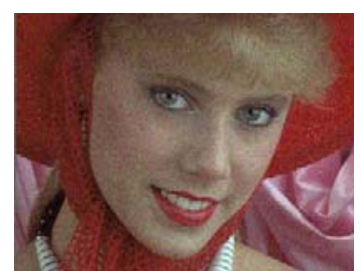

Fig. 10e Additive Gaussian noise in level 4

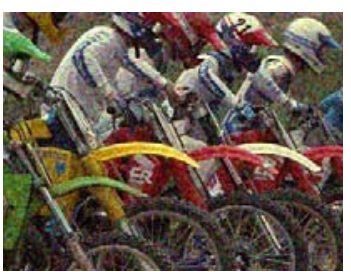

Fig. 11e Spatially correlated noise in level 4 
Table 8 Distortion types and considered subsets of the image database [28]

\begin{tabular}{|c|c|c|c|c|c|}
\hline & \multicolumn{5}{|c|}{ Image subsets } \\
\hline Type of distortion & Noise3 & JPEG & Exotic & Actual & Full \\
\hline Different additive noise in color components & - & - & - & - & + \\
\hline Masked noise & - & - & - & - & + \\
\hline High frequency noise & + & - & - & - & + \\
\hline Impulse noise & + & - & - & + & + \\
\hline Quantization noise & - & - & - & + & + \\
\hline JPEG compression & - & + & - & + & + \\
\hline JPEG2000 compression & - & + & - & + & + \\
\hline JPEG transmission errors & - & - & - & - & + \\
\hline JPEG2000 transmission errors & - & - & - & - & + \\
\hline Non eccentricity pattern noise & - & - & + & - & + \\
\hline Local blockwise distortions of different intensity & - & - & + & - & + \\
\hline
\end{tabular}

Number of samples of MOS data

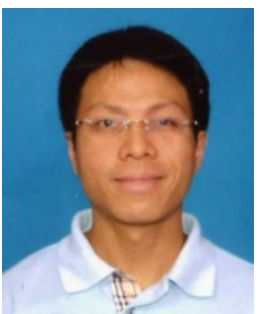

Kit Yan Chan received a $\mathrm{PhD}$ degree in Computing from London South Bank University, United Kingdom, in 2006. He is currently a Senior Lecturer in the Department of Electrical and Computer Engineering, Curtin University, Australia. He was the guest editor for Applied Soft Computing, Neurocomputing, Engineering Applications of Artificial Intelligence and International Journal of Fuzzy Systems (IJFS). He is the associate editor for Neurocomputing and IJFS, and the editorial board for Journal of Engineering Design. His research interests include machine learning perceptual quality evaluations and imprecise data mining.

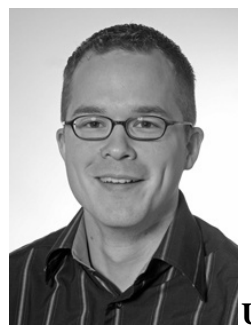

Ulrich Engelke (S'05-M'10-SM'13) received a $\mathrm{PhD}$ degree in Telecommunications from the Blekinge Institute of Technology, Sweden, in 2010. He is currently a Senior Research Scientist in Cognitive Informatics at the Commonwealth Scientific and Industrial Research Organisation (CSIRO) in Hobart, Australia. Ulrich is co-chair of the Psychophysiology-based Quality Assessment (PsyPhyQA) project of the Video Quality Experts Group (VQEG). His research interests are within quality of experience, visual analytics, and perceptual imaging. 